\title{
Global behaviour corresponding to the absolute instability of the rotating-disc boundary layer
}

\author{
By CHRISTOPHER DAVIES ${ }^{1}$ AND PETER W. CARPENTER 2 \\ ${ }^{1}$ School of Mathematics, Cardiff University, Cardiff, CF24 4YH, UK \\ ${ }^{2}$ School of Engineering, University of Warwick, Coventry, CV4 7AL, UK
}

(Received 6 April 2001 and in revised form 3 March 2003)

A study is carried out of the linear global behaviour corresponding to the absolute instability of the rotating-disc boundary layer. It is based on direct numerical simulations of the complete linearized Navier-Stokes equations obtained with the novel velocity-vorticity method described in Davies \& Carpenter (2001). As the equations are linear, they become separable with respect to the azimuthal coordinate, $\theta$. This permits us to simulate a single azimuthal mode. Impulse-like excitation is used throughout. This creates disturbances that take the form of wavepackets, initially containing a wide range of frequencies. When the real spatially inhomogeneous flow is approximated by a spatially homogeneous flow (the so-called parallel-flow approximation) the results of the simulations are fully in accordance with the theory of Lingwood (1995). If the flow parameters are such that her theory indicates convective behaviour the simulations clearly exhibit the same behaviour. And behaviour fully consistent with absolute instability is always found when the flow parameters lie within the theoretical absolutely unstable region. The numerical simulations of the actual inhomogeneous flow reproduce the behaviour seen in the experimental study of Lingwood (1996). In particular, there is close agreement between simulation and experiment for the ray paths traced out by the leading and trailing edges of the wavepackets. In absolutely unstable regions the short-term behaviour of the simulated disturbances exhibits strong temporal growth and upstream propagation. This is not sustained for longer times, however. The study suggests that convective behaviour eventually dominates at all the Reynolds numbers investigated, even for strongly absolutely unstable regions. Thus the absolute instability of the rotatingdisc boundary layer does not produce a linear amplified global mode as observed in many other flows. Instead the absolute instability seems to be associated with transient temporal growth, much like an algebraically growing disturbance. There is no evidence of the absolute instability giving rise to a global oscillator. The maximum growth rates found for the simulated disturbances in the spatially inhomogeneous flow are determined by the convective components and are little different in the absolutely unstable cases from the purely convectively unstable ones. In addition to the study of the global behaviour for the usual rigid-walled rotating disc, we also investigated the effect of replacing an annular region of the disc surface with a compliant wall. It was found that the compliant annulus had the effect of suppressing the transient temporal growth in the inboard (i.e. upstream) absolutely unstable region. As time progressed the upstream influence of the compliant region became more extensive. 


\section{Introduction}

For more than fifty years the rotating disc has been the model flow for studying three-dimensional boundary-layer instability and transition. During this period many papers have appeared describing theoretical and experimental research on this topic. One might have been forgiven, then, for assuming that the major features of instability and transition had already been discovered for this model flow and that only rather academic and arcane points remained to be elucidated. This view was to be dispelled by the revelation of Lingwood (1995) that the rotating-disc flow is absolutely unstable. Furthermore, the theoretical critical Reynolds number for absolute instability is more or less coincident with the experimentally observed transition point. A little later Lingwood (1996) also described an experimental investigation which appeared to corroborate her earlier theoretical study.

Absolute instability is a local concept in that it is defined theoretically by a stability analysis of the local velocity profiles. In effect, such an analysis assumes a spatially homogeneous flow. This is often termed the parallel-flow approximation. However, this term is, perhaps, not entirely appropriate for the rotating-disc flow as the boundary layer remains at constant thickness throughout. For this flow it is the increase in the magnitude of the undisturbed velocity field proportionately to radius that is responsible for the spatial inhomogeneity. In the present paper we investigate how spatial inhomogeneity affects the global response of this locally absolutely unstable flow. The study is based on direct numerical simulations of the complete linearized Navier-Stokes equations, obtained with the novel velocity-vorticity method presented in Davies \& Carpenter (2001, hereafter referred to as I).

It is important to emphasize that our numerical simulations are not equivalent to an instability analysis. In fact, they have more in common with physical experiments than stability theory. Beyond omitting the nonlinear terms in the form of the NavierStokes equations governing the perturbation flow field, the form of the perturbations in our simulations is not specified in any way. Just like a physical experiment the perturbations are initially excited by a local time-dependent displacement of the disc surface. The subsequent evolution of the disturbance is governed purely by the (linearized) Navier-Stokes equations. Accordingly, there is no need to evoke causality as in instability analysis, any more than one would need to do so for a physical experiment. Indeed, it would be inappropriate to attempt to do so.

Ours appears to be the first study of the global behaviour corresponding to the absolute instability of the rotating-disc boundary layer, $\dagger$ although, of course, Lingwood's experimental investigation is perforce also a study of the global behaviour. Previous numerical studies of the rotating-disc flow that took account of the inhomogeneous radial variation either did not reveal any vestige of absolute instability (Spalart 1991), or were based on the parabolic-stability-equation (PSE) approach (Malik \& Balakumar 1992) and therefore not able to accommodate the upstream propagation of disturbances.

Lingwood's discovery of the absolute instability was completely novel and came as a surprise to the research community. Nevertheless, as she herself pointed out, there were clues to be found in the previous experimental studies. (We will not attempt to review the relevant literature here, but instead refer the reader to the reviews by Reed \& Saric (1989) and Saric, Reed \& White (2003) and the introduction in Cooper \&

$\dagger$ During the revision of our paper we became aware of the analytical study of Peake \& Garrett (2003) which will be discussed below. 
Carpenter (1997a).) For example, in the surface flow visualizations of Gregory, Stuart \& Walker (1955), using the china-clay technique, the transitional radius is very sharp. More recent flow visualizations (e.g. Kobayashi, Kohama \& Takamadate 1980) also exhibit the same feature. The sharpness of the transition line on the rotating disc contrasts markedly with those normally found when boundary-layer transition is due to the amplification of convective instabilities like Tollmien-Schlichting waves. In these cases one typically sees wedges of turbulent flow upstream of the main transition line that are created by local roughness formed by the pigment or tracer particles used for the flow visualization. Lingwood (1995) also pointed out that the values of transitional Reynolds number measured in the many experimental studies of the rotating-disc flow differed by less than 3\% from the average value of about 513. Again, this is in sharp contrast with transition in flows dominated by convective instabilities, such as pipe flow or boundary layers over flat plates. In the latter case, for example, the transitional Reynolds number is very sensitive to background noise and environmental factors; consequently, transitional Reynolds numbers ranging from 100000 to in excess of $2 \times 10^{6}$ have been reported in the literature (Schlichting 1979).

Theoretical evidence pointing to the possibility of absolute instability was also available in the literature. First, as pointed out by Lingwood (1995), the velocity profiles for the rotating-disc boundary layer exhibit reverse flow when resolved in a range of directions between the radial and azimuthal directions. Since absolute instability requires upstream propagation, and a reverse undisturbed flow facilitates upstream propagation, it is found in many examples of absolutely unstable flow. Examples are given by Huerre \& Monkewitz (1990). These include countercurrent mixing layers in circular jets (Strykowski \& Niccum 1991), and the wakes behind circular cylinders (Mathis, Provansal \& Boyer 1984; Koch 1985; Triantafyllou, Triantafyllou \& Chryssostomidis 1986; Monkewitz 1988; Strykowski \& Sreenivasan 1990), blunt bodies (Hannemann \& Oertel 1989; Oertel 1990) and a floating cylinder (Triantafyllou \& Dimas 1989). But reverse flow certainly does not guarantee absolute instability, nor is it always necessary.

There were also more subtle theoretical indicators for absolute instability. It was known that the eigenspectrum for the local stability analysis of the rotating-disc boundary layer consisted of at least three distinct eigenmodes. Type I - the cross-flow vortices - was originally identified by Gregory et al. (1955) and is essentially inviscid. Type II is destabilized by Coriolis acceleration and is essentially viscous. It was originally discovered by Faller \& Kaylor (1966) (see also Faller 1991). Both types can exist in stationary and travelling form. A third eigenmode (Type III) was discovered by Mack (1985). The complexity of the eigenspectrum is relevant because for an absolute instability to occur two eigenmodes have to coalesce. In fact, Lingwood (1995) shows that Types I and III coalesce to form the absolute instability. This can, perhaps, be understood physically in terms of energy. The various eigenmodes (when distinct) can be classified as either positive- or negative-energy waves (PEW or NEW); see e.g. Cairns (1979). Irreversible energy transfer has a stabilizing effect on the PEW but a destabilizing effect on the NEW. Accordingly, one might expect that, owing to their opposite response to energy transfer, when a PEW and a NEW coalesce they become capable of being a true self-excited instability, rather than a noise amplifier like a convective instability. Cooper \& Carpenter (1997a) showed that Types I and II are NEW and PEW respectively. It is not clear whether or not Type III is a PEW, but presumably it is. Energy considerations suggest that Types I and II also could coalesce and form a self-excited instability. In fact, as shown by Lingwood (1995) and Cooper \& Carpenter (1997a), they do coalesce, but the 
result is an algebraically growing disturbance rather than an absolute instability. This phenomenon was investigated in I and also in a recent paper by Turkyilmazoglu \& Gajjar (2000).

Huerre \& Monkewitz (1990) give an excellent review of the global instability of spatially developing flows, especially its relationship to absolute instability. Accordingly, we will largely confine ourselves to reviewing the developments since 1990 that are relevant to our study. The concepts of absolute and convective instability are strictly only valid for a spatially homogeneous flow. They are extended to spatially developing flows by making a so-called quasi-parallel-flow approximation whereby one examines the stability of a model spatially homogeneous flow having the same streamwise velocity profile as the real spatially inhomogeneous flow at the selected spatial location. Thus a dispersion relation

$$
D(\alpha, \omega ; R)=0
$$

can be formulated linking the complex frequency and wavenumber at a given value of $R$ where $\alpha$ and $\omega$ are the non-dimensional wavenumber and frequency. $R$ is the Reynolds number based on the local spatial coordinate or variables. For example, for the rotating disc

$$
R=\frac{r_{r}^{*}}{\sqrt{v / \Omega}},
$$

where $r_{r}^{*}$ is the radial location where the stability analysis is carried out, $v$ is the kinematic viscosity, and $\Omega$ the rotational speed of the disc. Thus the coordinate at a fixed radial position is made dimensionless with reference to the constant boundary-layer displacement thickness. The same length scale is also used to make the wavenumber dimensionless and the reference time, $1 /(\Omega R)$, is used to make frequency dimensionless.

Purely in the context of boundary-layer instability over the rotating disc, the following remarks can be made about the use of (1). In the approach taken in classic linear instability theory one solves (1) for complex $\omega$, specifying $\alpha$ and keeping it real. Convective instability is then indicated when $\omega_{i}>0$ for a given combination of $\alpha_{r}$ and $R$. Gaster $(1962,1965)$ showed that spatially growing waves are a better representation of convectively unstable disturbances seen in most physical applications and experiments. In this case, $\omega$ is specified and kept real, and (1) is then solved for complex $\alpha$, and $-\alpha_{i}>0$ marks the onset of convective instability for a given combination of $\omega_{r}$ and $R$. A Gaster $(1962,1965)$ transformation can be used to obtain an approximate relationship between the temporal and spatial growth rates, namely

$$
-\alpha_{i} \simeq \frac{\omega_{i}}{c_{g}}
$$

where $c_{g}=\mathrm{d} \omega / \mathrm{d} \alpha$ is the local group velocity. So $\omega_{i}>0$ remains the criterion for convective instability. Evidently the Gaster transformation can only be used when the group velocity is finite (Gaster 1968), i.e. when a wavepacket would be convected away from its point of origin, as in figure 1(a). For absolute instabilities (figure $1 b$ ) there is true exponential temporal growth at a given spatial location. Plainly, the group velocity must be locally zero for absolute instability. However, this is not a sufficient condition (e.g. in the case of the rotating disc the group velocity is zero when the Types I and II eigenmodes coalesce, but the result is algebraic growth followed by convective instability, rather than absolute instability). In order to detect absolute instability one must allow both $\alpha$ and $\omega$ to be complex in the dispersion 

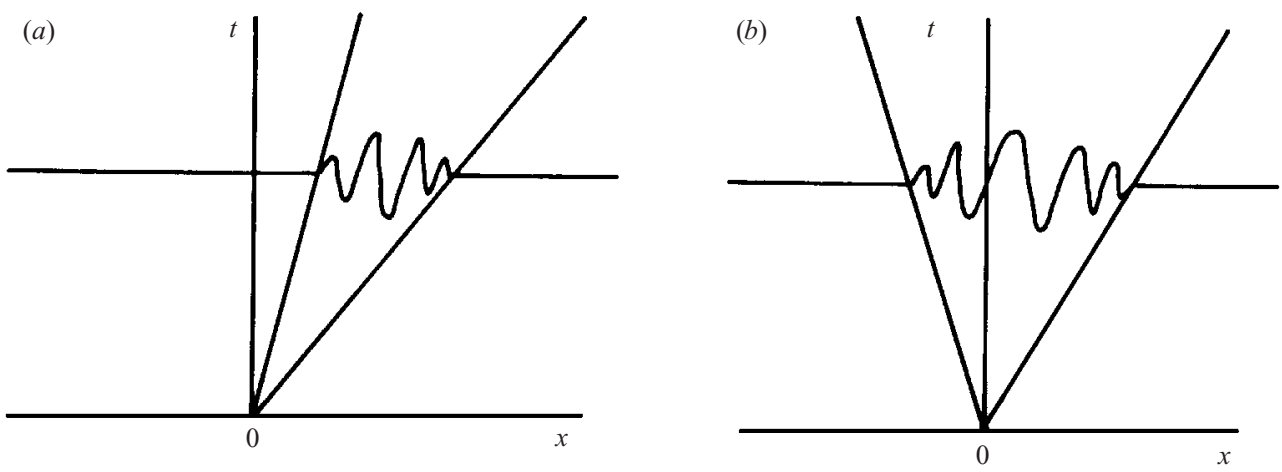

FIGURE 1. Schematic sketches of the evolution of a wavepacket generated by an impulse. (a) Convective instability. (b) Absolute instability.

relation (1), and use it to investigate the nature of the Green's function corresponding to the response of the flow to an impulse. Absolute instability is then indicated by the existence of a pinch-point in the complex $\alpha$-plane, as originally demonstrated by Briggs (1964) (see Huerre \& Monkewitz 1990; Lingwood 1995, 1997a). The value of $\omega_{i}$ corresponding to the pinch-point gives the non-dimensional absolute temporal growth rate, $\omega_{a, i}$.

In figure 2(a), we depict the characteristics of the convective and absolute instabilities for the rotating-disc flow. In this case both absolute and convective instability set in at distinct spatial locations. Formally the flow remains unstable for all $R>R_{c}$ (the critical Reynolds number for the onset of convective instability). According to Lingwood $(1995,1997 b)$ the critical value of the Reynolds number for convective instability is about 290 and that for absolute instability is $R_{a}=507.3$ (corrected in Lingwood $1997 b$ from the value of 510.6 given in Lingwood 1995). Furthermore, as shown in figure $2(a)$, the maximum values of both $\omega_{i}$ and $\omega_{a, i}$ are their asymptotic values as $R \rightarrow \infty$. The asymptotic value of $\left(\omega_{a, i}\right)_{\max }$ given in figure 2(a) is taken from Lingwood (1995). This apparently distinguishes it from the flows studied and reviewed by Huerre \& Monkewitz (1990) for which the maximum absolute growth rate occurred at finite values of $R$. For the rotating disc, one should include the real azimuthal wavenumber, $\beta$, as a parameter in (1) as well as $R$. For this reason the dependence of the region of absolute instability on both $R$ and $\beta$ is sketched schematically in figure $2(b)$. Figure $2(b)$ also plots the asymptotic absolute stability boundaries. The value for the upper branch, namely $\beta=0.265 R$, was given by the inviscid analysis of Lingwood (1995), whereas the lower branch, $\beta=0.026 R$ is given by the recent analysis of Peake \& Garrett (2003). Plainly, for a given $R$ the flow is only absolutely unstable for a range of values of $\beta$. (It should be noted that, in fact, only integer values of $\beta$ are physically admissible; but for simplicity $\beta$ is shown as continuously varying in figure $2 b$.)

In an important respect, however, the results presented in the previous paragraph, while formally correct, are misleading. This is because as $R \rightarrow \infty$, the azimuthal wavenumber corresponding to $\left(\omega_{a, i}\right)_{\max }$ varies as $\beta \simeq 0.1 R$. Thus the values of $\left(\omega_{a, i}\right)_{\max }$ plotted schematically in figure $2(a)$ do not correspond to fixed $\beta$. In fact, as pointed out by Peake \& Garrett (2003), the absolute growth rate for fixed $\beta$ not only exhibits a maximum, but only remains positive for a fixed range of $R$. This also follows from the finite range of $\beta$ corresponding to instability depicted in figure $2(b)$. The growth rate for constant $\beta$ is depicted as the short-dashed curve 

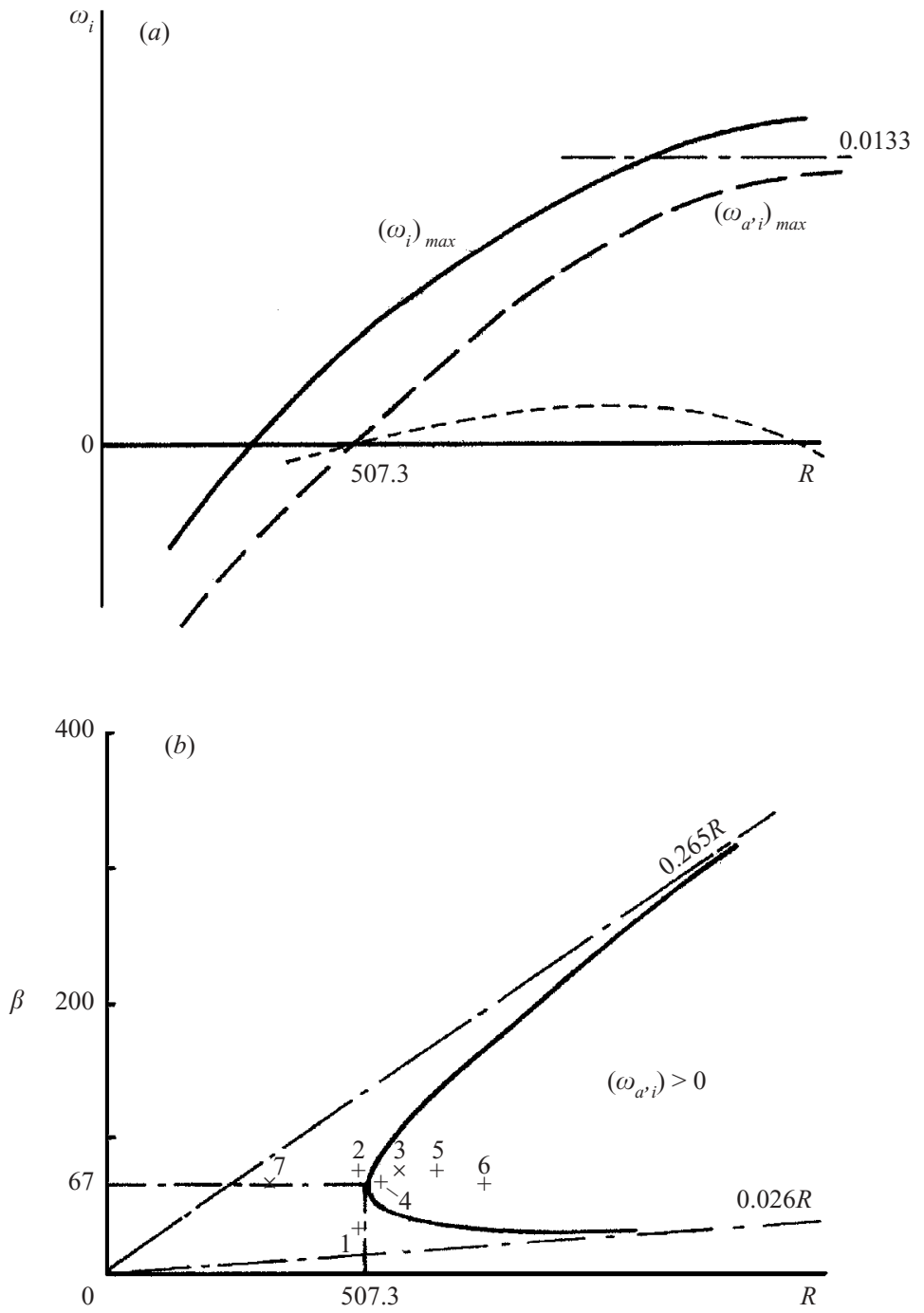

FIGURE 2. Schematic sketch depicting the characteristics of the local instability of the rotating-disc boundary layer. (a) Maximum growth rates vs. $R$; - - -, growth rate for fixed $\beta$. (b) Neutral curve defining the region of absolute instability; the labelled data points denote the conditions for the various numerical simulations presented in $\S 3$.

plotted in figure 2(a). Note that this curve is schematic only and does not provide a reliable guide to the magnitude of the maximum growth rate nor to the relative extent of the absolutely unstable region. Some idea of this can be deduced from the lower asymptote $\beta=0.026 R$ plotted in figure $2(b)$. From this we estimate that the absolutely unstable regime for the critical azimuthal wave-number, $\beta=67$, extends to $R \simeq 2600$.

Normally the region of absolute instability over the rotating disc is formally semiinfinite in extent in $R$. And even for constant $\beta$ absolute instability is found over a wide range of $R$. It is possible, however, as explained below, to make it finite and even relatively short, and therefore more similar to the cases discussed by Huerre \& Monkewitz (1990) and other authors. Cooper \& Carpenter (1997b) showed that only 
a slight degree of wall compliance is required to push the absolute instability much further outboard or to eliminate it entirely. Thus by making the disc surface compliant beginning at a radial location well into the absolutely unstable region, we can obtain a flow that is absolutely unstable in a relatively small finite region.

It was our intention at the outset of our study to address three key questions. First, is the existence of the semi-infinite region of absolute instability depicted in figure 2 associated with an amplified linear global response such that a typical flow variable

$$
A \sim \mathrm{e}^{-\mathrm{i} \omega_{G} t}
$$

(where $\omega_{G}=\omega_{G, r}+\mathrm{i} \omega_{G, i}$ is complex and $\omega_{G, i}>0$ )? Secondly, if it is, what is the value of $\omega_{G}$ and how is it determined? Lastly, in the case with a partially compliant surface, how large does the region of absolute instability need to be to elicit a linear global response of the form (3)? We investigated these questions by means of a numerical simulation of the complete, linearized, Navier-Stokes equations.

What guidance for investigating these questions do previous theoretical studies offer? The forced linearized Ginzburg-Landau equation, namely

$$
\frac{\partial A}{\partial t}+U \frac{\partial A}{\partial x}=\mu A+\gamma \frac{\partial^{2} A}{\partial x^{2}}+H(t) F(t) \delta(x),
$$

is one of the simplest systems that exhibits absolute instability. Here the system is forced at the point $x=0$ and the time variation of the forcing such that it is 'switched on' at $t=0$. The global instability of this equation with spatially inhomogeneous coefficients has been investigated by various authors. In such cases, for the purposes of theory the 'slow' spatial variable, $X=\epsilon x$ (where $\epsilon$ is a small parameter, typically $1 / R$ ) in a WKBJ approximation is regarded as complex. For example, Chomaz, Huerre \& Redekopp (1988) investigated the case of linearly varying $\mu=\mu_{0}+\mu_{1} x$ (where $\left.\mu_{1}<0\right)$. They established fundamental results that are central to the questions of how the existence of global modes and the selection of their complex frequency are connected to the local instability behaviour corresponding to $\mu=\mu_{0}=$ const. (See also Huerre \& Monkewitz 1990.) They examined the global behaviour for fixed $\mu_{1}$ as $\mu_{0}$ was increased from an initially negative value. Absolute instability occurs when $\mu_{0}$ exceeds the critical value $\mu_{a}$. But it was necessary for $\mu_{0}$ to exceed $\mu_{G}>\mu_{a}$ before the system became globally unstable. In other words the region of local absolute instability had to reach a certain threshold size before global instability ensued. (See, also, the WKBJ global stability analysis of the vorticity transport equation for non-parallel shear flow by Monkewitz, Huerre \& Chomaz 1993.) On the question of how the global frequency is selected, Huerre \& Monkewitz (1990) proved that $\omega_{G}$ corresponded to the frequency $\omega_{a}\left(X_{s}\right)$ of the absolutely unstable local mode located at a saddle point $X_{s}$ in complex $X$-space. This selection principle differs from early suggestions made by Pierrehumbert (1984), Koch (1985) and Monkewitz \& Nguyen (1987). It follows from the Huerre-Monkewitz selection principle that the global growth rate satisfies the following inequality:

$$
\omega_{G, i} \simeq \omega_{a, i}\left(X_{s}\right) \leqslant\left(\omega_{a, i}\right)_{\max } .
$$

This implies that a necessary, but not sufficient, condition for global instability is the existence locally of absolute instability. These principles have been rigorously proved by Chomaz, Huerre \& Redekopp (1991) for a class of Ginzburg-Landau equations within the WKBJ framework. Hunt \& Crighton (1991) also found that the frequencyselection principle is exact in their study of the global instability of the linearized Ginzburg-Landau equation. 
In the case of the rotating disc, the fact that $\partial\left(\omega_{a, i}\right)_{\max } / \partial R \rightarrow 0$ as $R \rightarrow \infty$ might seem to suggest that the equivalent of the saddle point $X_{s}$ used to determine the complex global frequency by Huerre \& Monkewitz is located at $R=\infty$. If this were so, then the Huerre-Monkewitz approach would be ill-posed in the case of the rotating disc. However, as pointed out above, for fixed $\beta$ the absolutely unstable region is finite in extent. This suggests that the saddle point is located at a finite value of $R$. Very recently, Peake \& Garrett (2003) have carried out an inviscid analysis of the global linear stability of the rotating-disc flow that shows the saddle point occurs at a finite value of $R$.

There have also been some experimental studies and numerical simulations of global instability. On the whole these are in accordance with the theoretical picture emerging from the studies of the Ginzburg-Landau equation. Most notable are the various experimental and numerical studies of the cylinder wake, reviewed by Huerre \& Monkewitz (1990) and briefly discussed above. The global instability of other wake flows has also been investigated by numerical simulation, e.g. the wakes behind blunt bodies (Hannemann \& Oertel 1989; Oertel 1990), shallow-water flow past bottom topography in the form of a circular bump (Schär \& Smith 1993), and the wake of a triangular cylinder (Zielinska \& Westfreid 1995). Certain jet and plume flows have also been found to exhibit global instability, e.g. hot jets (as reviewed by Huerre \& Monkewitz 1990), dripping taps (again, as reviewed by Huerre \& Monkewitz 1990, see also Le Dizès 1997), and, more recently, the flickering candle (Maxworthy 1999) and the plane wake flow with base suction (Leu \& Ho 2000). This last paper illustrates well how global instability can be eliminated by shrinking the region of absolute instability, here done by the application of base suction, which eliminates the global instability, even though it causes $\left(\omega_{a, i}\right)_{\max }$ to rise steeply. Moreover the level of base suction required to suppress the global instability is reasonably well predicted by the application of the WKBJ theory of Monkewitz et al. (1993).

Plainly, however, for our present investigation the most important experimental study is Lingwood (1996) that was aimed specifically at corroborating her theoretical discovery of absolute instability in the rotating-disc flow. It is immediately clear from her results that there is not a global response for the entire flow field. This had to be so, of course, otherwise the absolute instability would have been discovered much earlier. In her experiment the impulse excitation is triggered at a radial location, $R_{e}$, well inboard of $R_{a}$ (the critical value for absolute instability). For this case Lingwood proposed that the global response be as depicted in figure 3(a), where the wavepacket propagates initially as a convective instability, but its trailing edge turns as it approaches $R_{a}$ and tends towards a vertical line $R_{G}$, say, where $R_{G}$ is a little greater than $R_{a}$. She does not make any suggestions about other aspects of the global response. For example, does the flow downstream of $R=R_{G}$ possess the same complex global frequency (i.e. oscillate at the same frequency and grow at the same exponential rate)? This would seem somewhat unlikely in view of the clearly convective nature of the part of the wavepacket in the vicinity of its leading edge. Lingwood's experimental results (see her figure 15) are certainly consistent with the model depicted in figure $3(a)$. But, the main reason the experimental data carry conviction in supporting the view summarized in figure $3(a)$, is that they appear to corroborate her theory which, of course, is a purely local analysis. No evidence is available with regard to the existence of an amplified linear global response of the form (3). Nor is this to be expected, for, as Lingwood herself pointed out, in reality nonlinear effects would determine the final form of the global response. It is worth noting, perhaps, that the available theoretical and computational evidence on such 

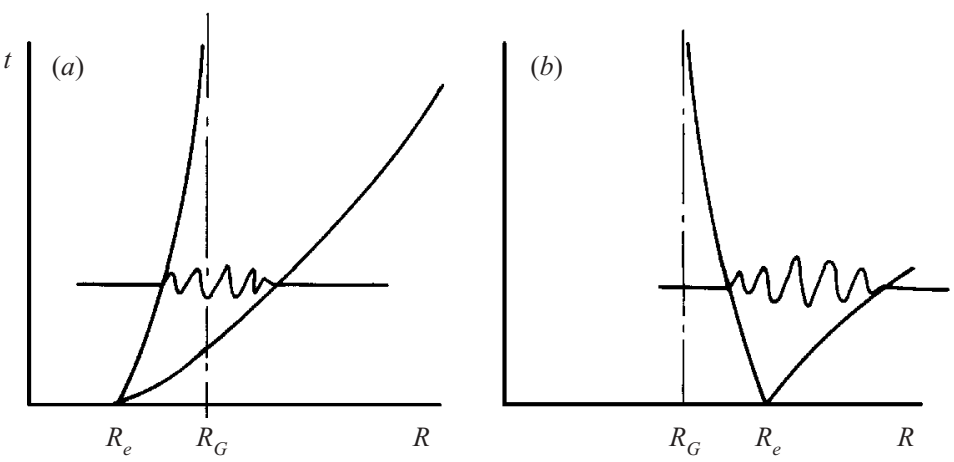

FIGURE 3. Typical wavepacket evolution for the rotating-disc boundary layer according to Lingwood's conjecture. Impulsive excitation $(a)$ at $R_{e}<R_{a}<R_{G}$ (based on figure $1 c$ of Lingwood 1996); and (b) at $R_{e}>R_{G}>R_{a}$.

nonlinear effects (e.g. Hannemann \& Oertel 1989; Zielinska \& Westfreid 1995; Pier \& Huerre 1996) suggests that the form of the linear global mode is qualitatively preserved, with nonlinear effects leading to saturation and in some cases causing the global frequency to rise slightly. The exception to this is the steep nonlinear global mode discovered by Pier et al. (1998) which may be more relevant in the present case.

In contrast, our numerical simulations presented herein suggest that the global behaviour of disturbances propagating in the rotating-disc boundary layer is dominated by convective instability at all radii (i.e. Reynolds numbers). There is no evidence of a persistent globally amplified mode of the form (3), indeed there is counter-evidence. The picture emerging from our study is best sumarized schematically in figure 4. Figure 4(a) shows the case when the boundary layer is forced impulsively in the region of convective instability. It corresponds to Lingwood's experiment and to her interpretation shown in figure $3(a)$. Figure $4(a)$ is quite similar to figure $3(a)$ and consistent with Lingwood's experimental data. However, the trailing edge of the wavepacket shows no sign of asymptoting to a vertical line anywhere near the critical value of $R_{a} \simeq 507$. Moreover, we give additional evidence showing that the long-term behaviour is convective. A numerical simulation has the advantage compared with an experiment that it is feasible to place the impulsive excitation at a location well beyond $R_{a}$. This would correspond to figure $3(b)$. If an amplified global mode exists, this arrangement would surely be more likely to reveal it than the one corresponding to figure 4(a). Figure 4(b) shows schematically what we believe our simulations imply about both the short- and long-time behaviour. Initially, the wavepacket behaves in a manner consistent with an absolute instability and strong local temporal growth occurs as well as upstream propagation. This is not sustained, however; it seems that once the trailing edge of the wavepacket reaches a position near $R=R_{a}$, it begins to turn and eventually reverses direction. Ultimately at large times the wavepacket behaves convectively. The situation seems analogous to algebraic growth in that there is strong temporal growth locally for a short duration, presumably strong enough for nonlinear effects to enter and lead to transition. Like the algebraic growth investigated in I, ultimately it is dominated by the strong convective instability. In many ways, it is the reverse of the scenario described by Huerre \& Monkewitz (1990) and found in the cylinder wake, for example, whereby a region of absolute instability acts as a global oscillator and dominates the downstream region of nominally convective instability. 

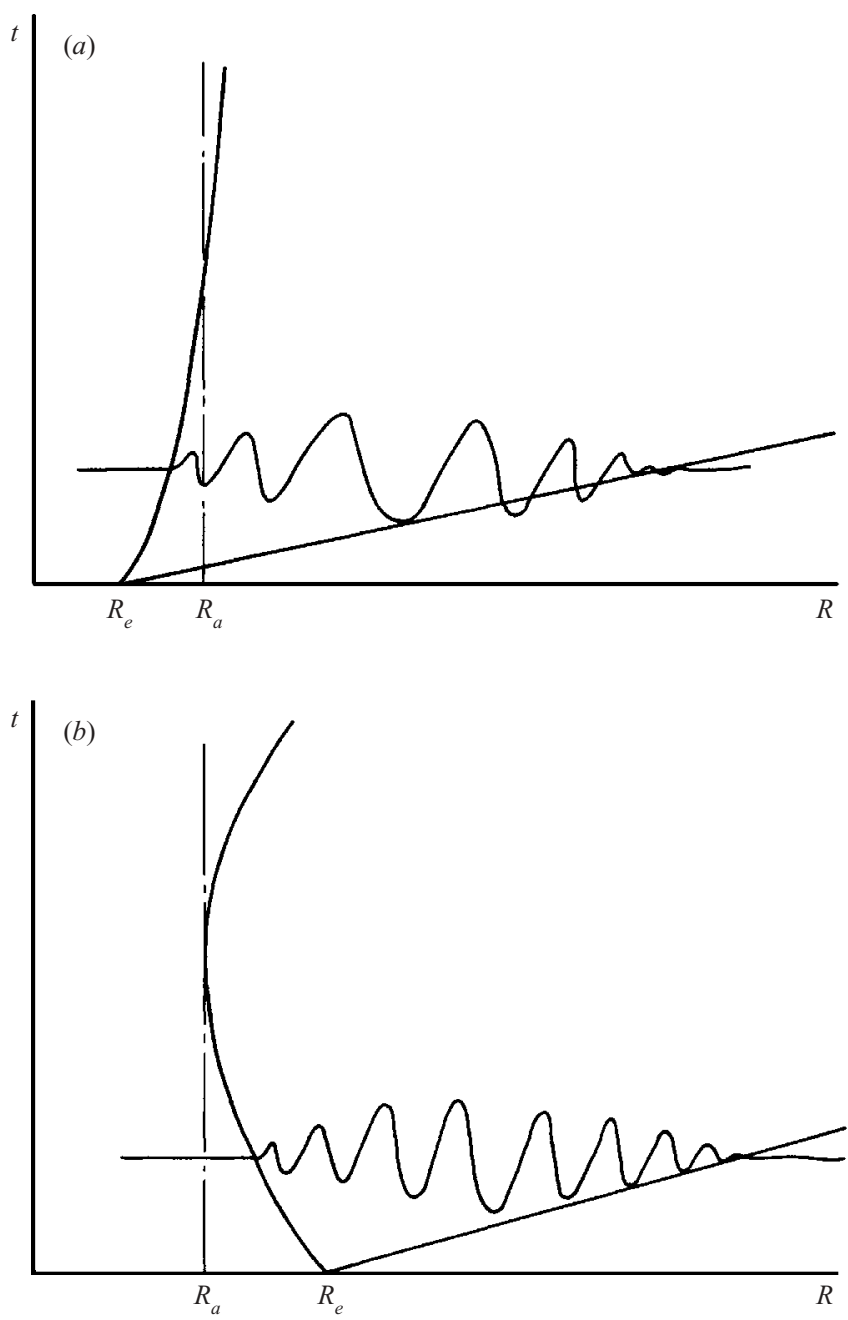

FIGURE 4. Schematic sketches of typical wavepacket evolution for the rotating-disc boundary layer as revealed by our numerical simulations. Impulsive excitation $(a)$ at $R_{e}<R_{a}$; and (b) at $R_{e}>R_{a}$.

For the rotating disc the upstream convective behaviour appears to be so strong that it dominates the extensive downstream region of absolute instability.

Our results do not seem to conform with the previous work on the global behaviour associated with absolute instability. Evidence from two recent sources, however, strongly suggests that the global response found in our study is not anomalous. First, Monkewitz (2001, personal communication) points out that the behaviour depicted in figure $4(b)$, whereby the trailing edge of the evolving wavepacket turns back on itself, can be reproduced qualitatively by the linearized Ginzburg-Landau equation (4). All that is required is for the growth rate in (4) to vary as $\mu=\mu_{0}+\mu_{1} X$ with complex coefficients such that $\operatorname{Re}\left(\mu_{1}\right)>0$. This corresponds qualitatively, more or less, with the rotating-disc characteristics illustrated schematically in figure $2(a)$. Hunt \& Crighton (1991) did not consider this case with complex $\mu$ explicitly. But, as Monkewitz explains, the generic behaviour referred to above can be deduced fairly easily from the exact Green's function (equation 31 of Hunt \& Crighton). He further explains that 
the reason why this behaviour only occurs for complex $\mu_{1}$ in the Ginzburg-Landau model is intuitively understandable, since the downstream edge reaches more and more 'oscillators' at frequencies that are increasingly different (without bound) from the absolute frequency at any given spatial location.

The other recent evidence that supports the results of our study is due to Peake \& Garrett (2003). They carried out a global, linear, inviscid, stability analysis of the boundary layer on rotating bodies including the rotating disc as a special case. They find the saddle point that plays a crucial role in the Huerre-Monkewitz approach is located at a finite (complex) value of non-dimensional radius. And, even more significantly, they find that the linear global mode is damped, in agreement with the results of our numerical-simulation study. These results may not be in conflict with the inequality (5) and other principles laid out by Huerre \& Monkewitz (1990) and subsequent workers. It is just that for the rotating disc even a semi-infinite region (finite, but extensive, for fixed $\beta$ ) of absolute instability is insufficient for an amplified global response.

The remainder of the paper is set out as follows. The theoretical and numerical approach has been presented in detail in I, so only fairly brief descriptions are given in $\S 2$. Section 3 is the main section of the paper. Here we present the results of our numerical-simulation study of the global behaviour of impulsively generated disturbances in the boundary layer of a rigid rotating disc. We study both cases which are convectively unstable in the equivalent spatially homogeneous flow and those that are absolutely unstable. In $\S 4$ we investigate the effects of replacing part of the rigid disc in the absolutely unstable region with a compliant annulus. Finally, conclusions are given in $\S 5$.

\section{Theoretical and numerical approach}

The theoretical and numerical approach is fully described in I. Accordingly, only an outline will be given here.

For the rotating disc the dimensional flow field is defined with respect to a cylindrical coordinate system $\left(r^{*}, \theta, z^{*}\right)$ that is fixed relative to the disc which rotates at speed $\Omega$. The asterisks denote dimensional length coordinates. The undisturbed flow field in this coordinate system is given by the dimensional velocity vector

$$
\boldsymbol{U}^{*}=\left(U_{r}^{*}, U_{\theta}^{*}, W\right) .
$$

These velocity components are related to the von Kármán (1921) similarity solution $(F(z), G(z), H(z))$ (where $z=z^{*} \sqrt{\Omega / v}$ and $v$ is the kinematic viscosity of the fluid) for the rotating-disc boundary layer as follows:

$$
U_{r}^{*}=r^{*} \Omega F(z), \quad U_{\theta}^{*}=r^{*} \Omega G(z), \quad W=\sqrt{\nu \Omega} H(z) .
$$

Thus the boundary-layer displacement thickness, $\delta^{*}=(v / \Omega)^{1 / 2}$, is used as the reference length scale for non-dimensionalization; but we also introduce another length scale, namely $r_{r}^{*}-$ a reference value of the radial coordinate. Thus $r=r^{*} / \delta^{*}$ and the Reynolds number is defined as $R=r_{r}^{*} / \delta^{*} ; r_{r}^{*} \Omega$ is used as the reference velocity, $1 /(R \Omega)$ as reference time, $R \Omega$ as the reference vorticity and $\rho r_{r}^{* 2} \Omega^{2}$ as the reference pressure. Note that there is some slight variation in notation from I where $\Omega$ was used for undisturbed vorticity rather than rotation speed as here. 
Perturbations to the velocity and vorticity fields are introduced that in nondimensional form are denoted by

$$
\boldsymbol{u}=\left(u_{r}, u_{\theta}, w\right), \quad \boldsymbol{\omega}=\left(\omega_{r}, \omega_{\theta}, \omega_{z}\right) .
$$

It was shown in I that, subject to rather general conditions as $z \rightarrow \infty$ that are definitely satisfied in the present case, the Navier-Stokes equations are fully equivalent the following system of governing equations for the primary variables $\left(\omega_{r}, \omega_{\theta}, w\right)$ :

$$
\begin{gathered}
\frac{\partial \omega_{r}}{\partial t}+\frac{1}{r} \frac{\partial N_{r}}{\partial \theta}-\frac{\partial N_{\theta}}{\partial z}-\frac{2}{R}\left(\omega_{\theta}+\frac{\partial w}{\partial r}\right)=\frac{1}{R}\left\{\left(\nabla^{2}-\frac{1}{r^{2}}\right) \omega_{r}-\frac{2}{r^{2}} \frac{\partial \omega_{\theta}}{\partial \theta}\right\}, \\
\frac{\partial \omega_{\theta}}{\partial t}+\frac{\partial N_{r}}{\partial z}-\frac{\partial N_{z}}{\partial r}+\frac{2}{R}\left(\omega_{r}-\frac{1}{r} \frac{\partial w}{\partial \theta}\right)=\frac{1}{R}\left\{\left(\nabla^{2}-\frac{1}{r^{2}}\right) \omega_{\theta}+\frac{2}{r^{2}} \frac{\partial \omega_{r}}{\partial \theta}\right\}, \\
\nabla^{2} w=\frac{1}{r}\left(\frac{\partial \omega_{r}}{\partial \theta}-\frac{\partial\left(r \omega_{\theta}\right)}{\partial r}\right) .
\end{gathered}
$$

where

$$
\boldsymbol{N}=\left(N_{r}, N_{\theta}, N_{z}\right)=(\nabla \times \boldsymbol{U}) \times \boldsymbol{u}+\boldsymbol{\omega} \times \boldsymbol{U}+\underbrace{\boldsymbol{\omega} \times \boldsymbol{u}}_{\mathrm{nl}} .
$$

The secondary variables are defined in terms of the primary ones as follows:

$$
\begin{gathered}
u_{r}=-\int_{z}^{\infty}\left(\omega_{\theta}+\frac{\partial w}{\partial r}\right) \mathrm{d} z, \\
u_{\theta}=\int_{z}^{\infty}\left(\omega_{r}-\frac{1}{r} \frac{\partial w}{\partial \theta}\right) \mathrm{d} z, \\
\omega_{z}=\frac{1}{r} \int_{z}^{\infty}\left(\frac{\partial\left(r \omega_{r}\right)}{\partial r}+\frac{\partial \omega_{\theta}}{\partial \theta}\right) \mathrm{d} z .
\end{gathered}
$$

Only the perturbations are to be determined in the numerical simulation. In the present study we are only interested in disturbances with vanishingly small amplitude, so we linearize the governing equations by simply omitting the term labelled $\mathrm{nl}$ in (12). The resulting system of equations is fully equivalent to the complete linearized Navier-Stokes equations. The linearization permits the boundary conditions at the wall to be simplified in the case when the wall moves in the vertical direction. Vertical displacement of the wall occurs when the wall is compliant and also in the vicinity of the perturbation exciter or driver. The linearized no-slip conditions and the wall-normal zero-displacement conditions become

$$
u_{r}=-\frac{r}{R} F^{\prime}(0) \eta, \quad u_{\theta}=-\frac{r}{R} G^{\prime}(0) \eta, \quad w=\frac{\partial \eta}{\partial t} \quad \text { at } \quad z=0
$$

$(16 a, b, c)$

where $\eta$ is the non-dimensional vertical wall displacement. The wall displacement equals zero for a rigid wall. Otherwise it is either specified, as in the case of the driver, or governed by the equation of motion for the compliant wall (see I). Substituting (16a) and (16b) into the definitions (13) and (14) for the secondary variables gives the following integral constraints on the primary variables which replace the no-slip conditions (16a) and (16b):

$$
\int_{0}^{\infty} \omega_{\theta} \mathrm{d} z=\frac{r}{R} F^{\prime}(0) \eta-\int_{0}^{\infty} \frac{\partial w}{\partial r} \mathrm{~d} z,
$$




$$
\int_{0}^{\infty} \omega_{r} \mathrm{~d} z=-\frac{r}{R} G^{\prime}(0) \eta+\int_{0}^{\infty} \frac{1}{r} \frac{\partial w}{\partial \theta} \mathrm{d} z
$$

Equation $(16 c)$ in its original form as a boundary condition acts as the third constraint on a primary variable.

Linearization makes the problem separable with respect to the azimuthal coordinate $\theta$, which is particularly convenient given our use of a Fourier spectral representation to discretize the azimuthal variation. Thus any given perturbation can be represented as a linear superposition of independent azimuthal modes. We can therefore independently calculate individual azimuthal modes of the perturbation velocity and vorticity fields that take the form

$$
\left(u_{r}, u_{\theta}, w\right)=\left(\hat{u}_{r}, \hat{u}_{\theta}, \hat{w}\right) \mathrm{e}^{\mathrm{i} n \theta}, \quad\left(\omega_{r}, \omega_{\theta}, \omega_{z}\right)=\left(\hat{\omega}_{r}, \hat{\omega}_{\theta}, \hat{\omega}_{z}\right) \mathrm{e}^{\mathrm{i} n \theta}
$$

where $\hat{u}_{r}$ etc. are functions only of $r$ and $z$, and $n$ is the integer-valued azimuthal mode number (this notation that recognizes explicitly the integer value replaces $\beta$ used in $\S 1$ where, for convenience, the azimuthal wave-number was regarded as a real number - see figure $2 b$ ). The remaining notation is conventional and should be clear. All coordinates and flow quantities are non-dimensional unless specified otherwise. Equation (19) is an inevitable consequence of linearization and not an additional assumption. No other assumptions are made about the form of the perturbations.

As described in I, a fourth-order, centred, compact finite-difference scheme is used for discretization in the $r$-direction, a Chebyshev spectral scheme used in the wallnormal, i.e. $z$-direction, and Fourier spectral scheme used in the azimuthal direction. As explained above, for a linear simulation, this form of azimuthal discretization permits us to treat each azimuthal mode independently. Some of the simulations reported below were also repeated using higher-order finite-difference schemes in order to check that the phase errors with the fourth-order scheme were not significant.

All perturbation quantities are set equivalent to zero at the radial inflow boundary. Various strategies for dealing with the outflow boundary condition are described in I. For the numerical simulations described herein, that are particularly sensitive because of the issue of absolute instability and possible global instability, we always took care to assess whether there were any spurious effects that could be attributed to the outflow (i.e. outer) radial boundary. Whenever possible, we ensured that the outflow boundary remained well ahead of any disturbance with a discernible magnitude. However, for some simulations it was necessary to undertake very long time integrations in order to identify with confidence the qualitative form of the disturbance evolution. As a consequence, even with the use of an extremely long computational domain, e.g. $r_{\text {outer }}-r_{\text {inner }} \simeq 2500$, it was not possible to ensure that the disturbance amplitude always remained negligible at the outer radial boundary. In such cases strict tests were carried out to confirm that, within the relatively small region of interest where we were investigating the form of the disturbance in detail, there was no contamination of the perturbation flow field due to spurious transmission effects being fed back from the outer computational boundary. In practice, such checking involved the repetition of simulations using computational domains of increasing radial extent. Additional details are given in the Appendix. Anticipating the discussion of our results, it is interesting to note that when the radial extent of the computational domain was insufficient to avoid unwanted feedback, this was found to lead to spurious temporal growth. 


\section{Numerical simulations for rigid rotating discs}

In I we investigated disturbances excited by time-periodic motion of the surface. We now consider the development of disturbances generated by a localized impulsive wall motion. The wall displacement $\eta$ is taken to be of the form

$$
\eta(r, \theta, t)=a(\delta r) b(t) \mathrm{e}^{\mathrm{i} n \theta}, \quad \delta r=r-r_{e},
$$

with the temporal impulse given by

$$
b(t)=\left(1-\mathrm{e}^{-\sigma t^{2}}\right) \mathrm{e}^{-\sigma t^{2}} .
$$

Typical forms for the function $a$ used to localize the forcing motion around a given radius $r=r_{e}$ were described in $\mathrm{I}$. As before, $n$ is the azimuthal mode number. The parameter $\sigma$, which fixes the duration of the impulse, was chosen to be large enough for a broad range of temporal frequencies to be incorporated in the excitation. Its magnitude, and hence the highest effective frequency excited, was limited only by the need to retain full temporal resolution of the impulse.

Numerical simulations were conducted first with the fluid governing equations simplified according to the so-called parallel-flow approximation, i.e. the equations were made spatially homogeneous. $\dagger$ Results from these simulations were found to conform with the theory of Lingwood (1995). In particular, the existence of absolute instability could be inferred from the temporal behaviour of the simulated disturbances at Reynolds numbers and azimuthal mode numbers lying within the absolutely unstable region determined by Lingwood (see figure $2 b$ ). In order to keep our exposition concise, we defer until later any further discussion of the results obtained with the approximate, $\mathrm{SH}$, governing equations. Attention will instead be focused on the behaviour of disturbances that evolve in the real SI boundary layer.

\section{Convective instability}

Figure 5 shows time histories, at successive radial locations, for a disturbance with azimuthal mode number $n=32$ triggered by an impulse centred at $r_{e}=500$. (This combination is denoted by Point 1 in figure $2 b$.) The azimuthal component of the vorticity $\omega_{\theta w}$ at the wall is plotted for a fixed value of $\theta$, along with the corresponding envelopes $\pm\left|\omega_{\theta w}\right|$ obtained from the complex-valued amplitude. (No particular significance should be attached to our repeated selection of $\omega_{\theta w}$ as a convenient flow-field variable when we discuss the evolution of disturbances. The description of disturbance behaviour, particularly with regard to distinctions between absolute and convective instability, would not need to be altered in any fundamental, qualitative, manner if some other flow-field variable, such as the disturbance energy, were monitored instead of $\omega_{\theta w}$. However, as would be expected, the mean-flow SI has the effect of making any calculation of spatial growth rates dependent, to some extent, on the variable selected for measuring the disturbance amplitude. Since we are mostly concerned with detecting the presence or absence of temporal growth, such subtleties need not detain us any further here.)

From figure 5(a), it can be seen that the disturbance decays rapidly at $r=r_{e}$. For $r>r_{e}$, there is an initial period of quiescence while the disturbance propagates towards the given location and away from its source. As would be expected, the length of this quiescent time interval increases with the radius. When the disturbance eventually

$\dagger$ Hereafter the abbreviations SH and SI will be used respectively for spatially homogeneous and spatially inhomogeneous (or spatial inhomogeneity). 

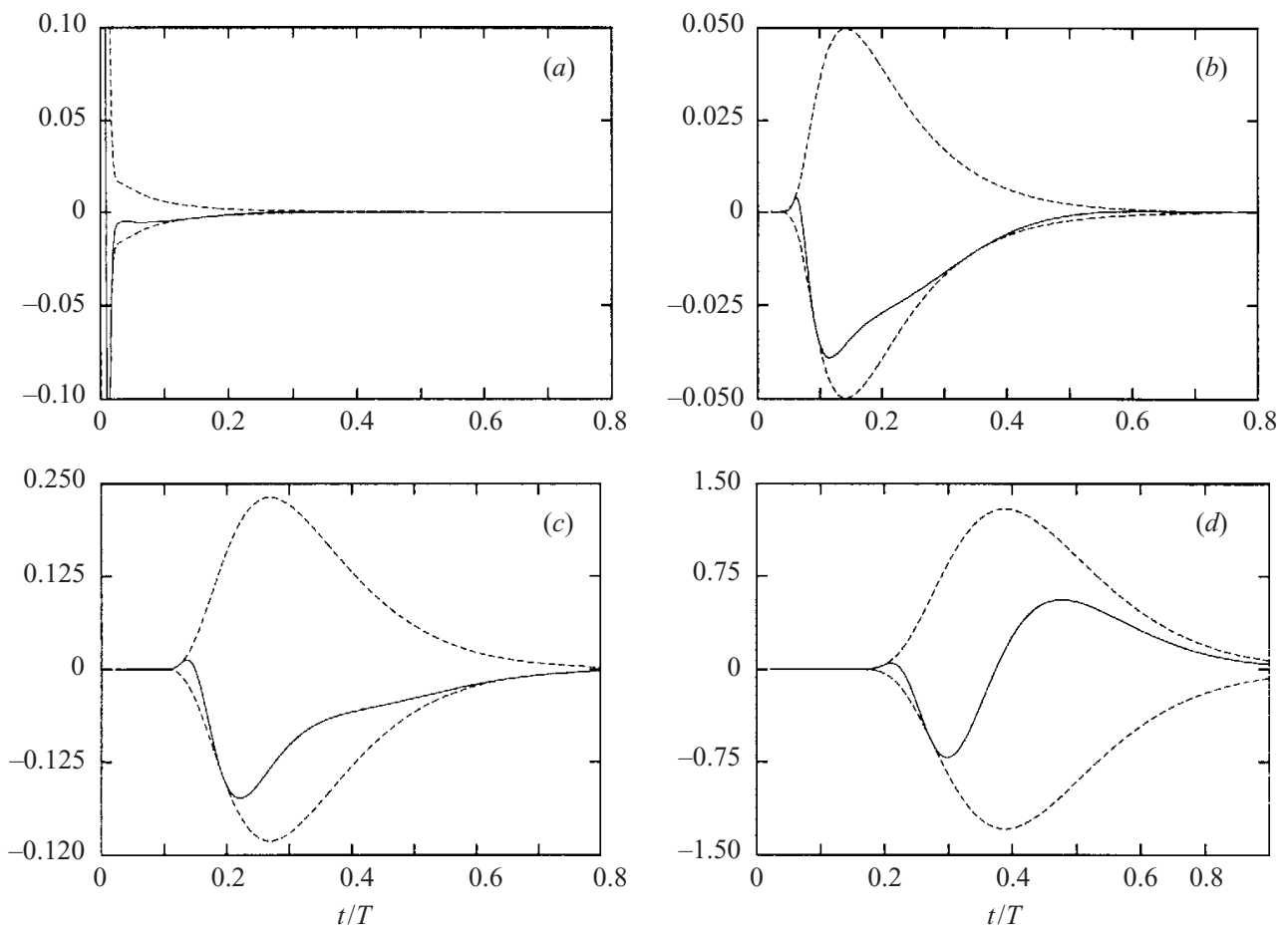

FIGURE 5. Time histories for $\omega_{\theta w}$ (solid lines), together with corresponding envelopes $\pm\left|\omega_{\theta w}\right|$ (broken lines), for an impulsively excited disturbance. $n=32$ and $r_{e}=500$. (a) $r=r_{e}=500$, (b) $r=525$, (c) $r=550$, (d) $r=575 . T=2 \pi R$ is the non-dimensional time period for the disc rotation.

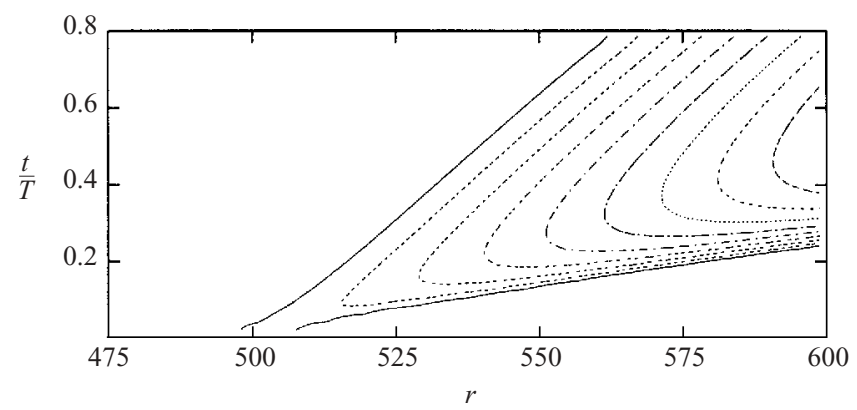

FIGURE 6. Spatio-temporal development of $\left|\omega_{\theta w}\right|$ for an impulsively excited disturbance with $n=32$. The impulsive disc surface motion used to excite the disturbance was centred at $r_{e}=500$. (The contours are drawn using a logarithmic scale with levels $A 2^{m}$ for $m=0,1,2 \ldots$, where $A$ is an arbitrary normalization factor.)

reaches each of the indicated radial positions, there is a period of growth followed by a relatively slow decay. Such behaviour is clearly indicative of the convective nature of the disturbance. That the flow is strongly unstable is evident from the radial growth in the disturbance apparent in figure 5 when account is taken of the different scales used for the axes in the separate plots. The convectively unstable character of the disturbance is even more plain in the spatio-temporal contour plots of $\left|\omega_{\theta w}\right|$ that are displayed in figure 6 . It is possible to identify both the leading and trailing 

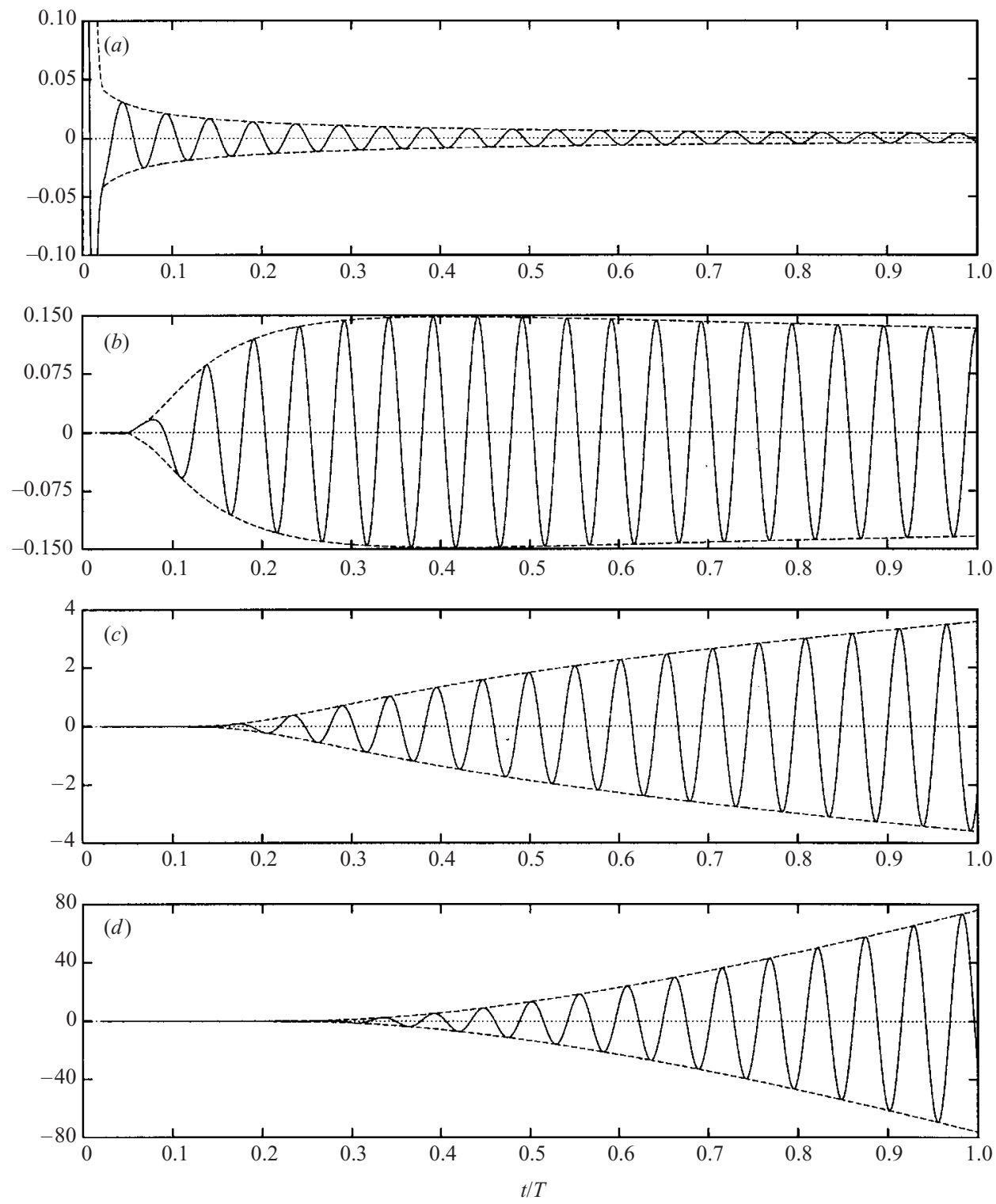

FIGURE 7. Time histories for $\omega_{\theta w}$ (solid lines), together with corresponding envelopes $\pm\left|\omega_{\theta w}\right|$ (broken lines), for a disturbance with $n=75$ that is impulsively excited at $r_{e}=500$. (a) $r=r_{e}=500,(b) r=525$, (c) $r=550$, (d) $r=575$.

edges of a radially extended disturbance wavepacket. Both ends of the wavepacket propagate outwards with non-zero radial velocities, the leading edge travelling rather more quickly than the trailing edge.

Disturbances that are absolutely unstable according to a local SH analysis We next consider an impulsively excited disturbance with azimuthal mode number $n=75$. According to Lingwood's (1995) local SH analysis this disturbance should be absolutely unstable for $r$ greater than approximately 520. Figure 7 presents the temporal development of the disturbance at successive radial locations. As before, 


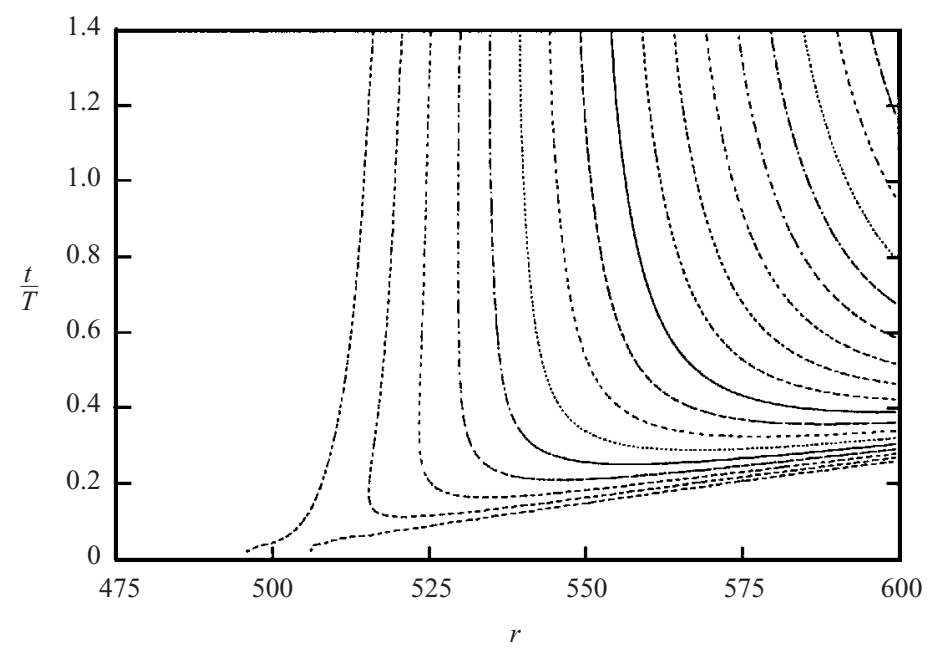

FIGURE 8. Spatio-temporal development of $\left|\omega_{\theta w}\right|$ for an impulsively excited disturbance with $n=75$. The impulsive disc surface motion used to excite the disturbance was centred at $r_{e}=500$. (Contours drawn using a logarithmic scale, as in figure 6.)

excitation was located at $r_{e}=500$. (See Point 2 in figure $2 b$.) It can be seen that the disturbance still decays at $r=r_{e}$, but much less rapidly than for $n=32$. At $r=525$ there is a period of growth followed by a relatively weak decay. Further out, at $r=550$ and $r=575$, the disturbance exhibits continuous growth over the whole of the time interval considered. This behaviour provides a marked contrast with the brief growth, followed by decay, exhibited at the corresponding radial positions when $n=32$.

The spatio-temporal development of the disturbance with $n=75$ is also displayed in figure 8, using contour plots of $\left|\omega_{\theta w}\right|$. It may be seen that the leading edge propagates radially outwards with a non-zero velocity comparable to that found when $n=32$. However, the trailing edge behaviour is very different than found previously. From a comparison with figure 6 , it appears that the trailing edge propagates much more slowly for $n=75$ than for $n=32$. In fact, the contour plots suggest that the radial velocity of the trailing edge may be approaching zero as the disturbance develops. Such behaviour would be expected if the disturbance exhibited persistent temporal growth at sufficiently large radii. Examination of the numerical simulation data confirmed that, for the time interval of the simulation, there was continual growth for all radial positions beyond $r \simeq 535$. This result may be checked by undertaking a graphical analysis of the contours displayed in figure 8. Locations where there is temporal growth can be determined from the intersections between the contours and vertical lines drawn at each selected radial position.

A more detailed examination of the simulation data plotted in figure 8 revealed that the temporal growth of the disturbance at any fixed radial position was much weaker than the convective growth occurring along the trajectory of the maximum of the wavepacket amplitude. For instance, at $r=550$ the disturbance amplitude, as measured by $\omega_{\theta w}$, grows by a factor $O\left(2^{3}\right)$ between $t=0.2 T$ and $t=0.4 T$, where $T=2 \pi R$ is the non-dimensional time period for the disc rotation. The amplitude then grows more weakly, by a further factor less than 2, between $t=0.4 T$ and $t=0.6 T$. Over the first of these two time intervals the location of the maximum amplitude of the disturbance wavepacket shifts radially outwards from $r \simeq 545$ to $r \simeq 600$ and the 

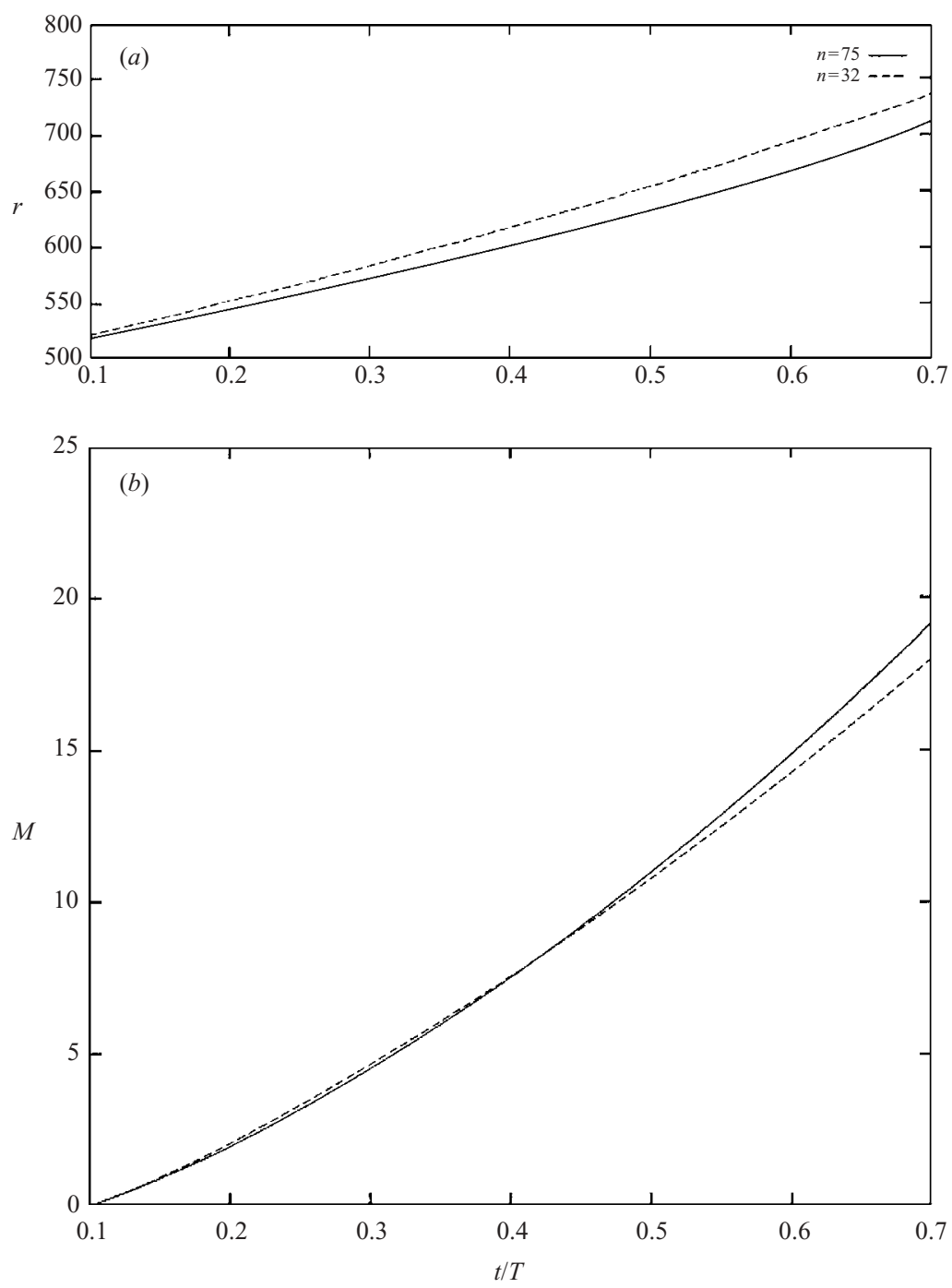

FIGURE 9. Development of the maximum value of $\left|\omega_{\theta w}\right|$ for disturbance wavepackets generated by impulsive excitation centred about $r_{e}=500$ for $n=75$ and $n=32$. (a) Radial trajectories of the amplitude maxima; $(b)$ magnitudes of the maxima plotted on a logarithmic scale. For both $n=75$ and $n=32$ the magnitudes have been normalized so that they are equal to unity for $t=0.1 T$. With such a normalization, the quantity displayed in $(b)$ is defined to be $M=\log _{2}\left|\omega_{\theta w}\right|_{\max }$ where the maximum is taken over all radial locations for each time instant.

magnitude of the maximum grows by a comparatively large factor $O\left(2^{6}\right)$. During the second of the stated time intervals the maximum disturbance amplitude grows by a still larger factor $O\left(2^{7}\right)$ and moves out to $r \simeq 665$. Figure 9 displays the trajectory and amplification of the wavepacket maximum, both for $n=75$ and $n=32$. From the plots of the trajectories it may be seen that the maximum in the disturbance wavepacket amplitude propagates outwards slightly more quickly for $n=32$ than for $n=75$, but otherwise the paths taken are quite similar. Thus the disparity that was noted between the radial velocities of the trailing edges is not reflected in the radial propagation of the wavepacket maxima. Further similarities in the evolution of the 

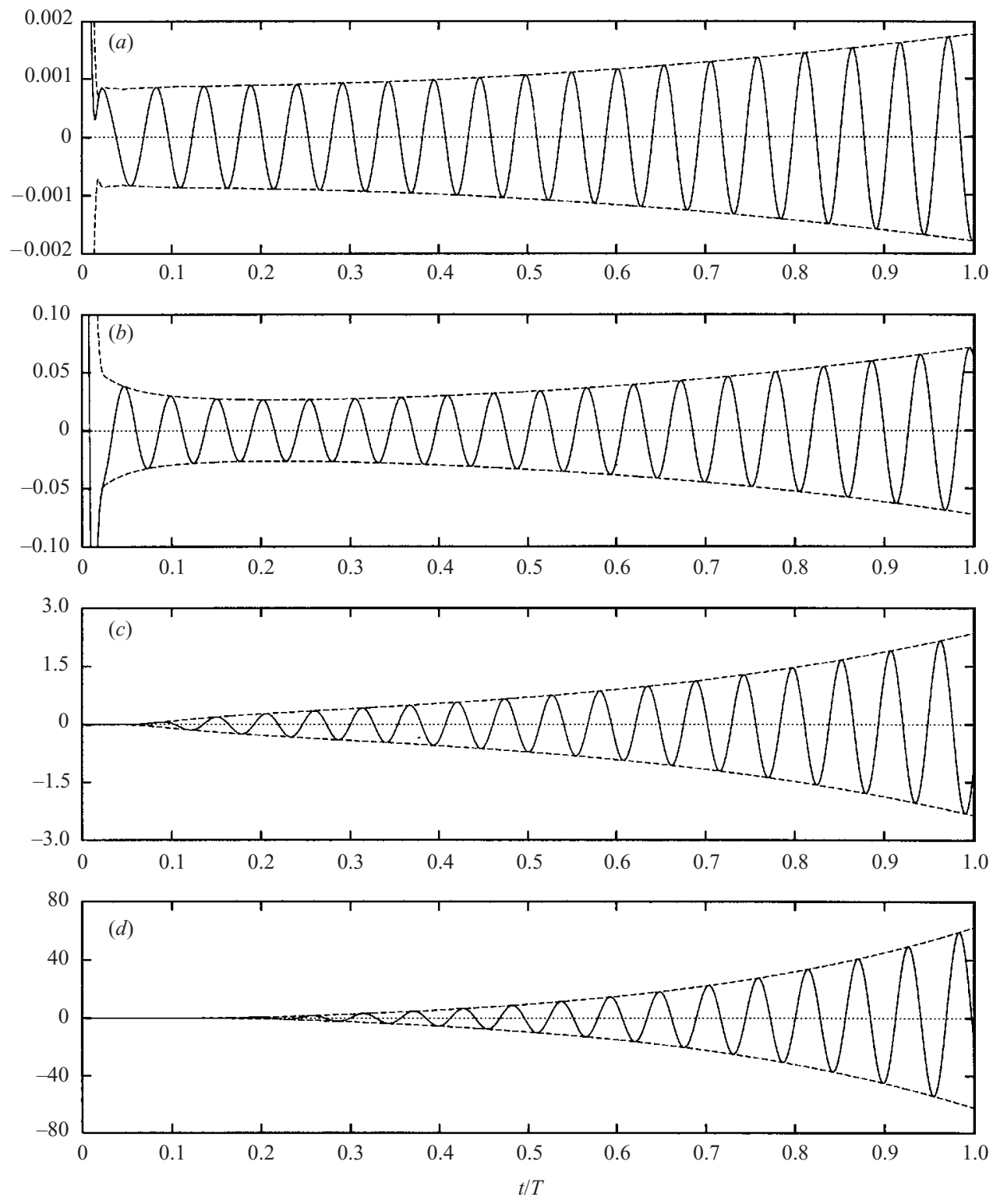

FiguRE 10. Time histories for $\omega_{\theta w}$ (solid lines), together with corresponding envelopes $\pm\left|\omega_{\theta w}\right|$ (broken lines), for a disturbance with $n=75$ that is impulsively excited at $r_{e}=550$. (a) $r=525$; (b) $r=r_{e}=550 ;(c) r=575 ;(d) r=600$.

disturbance wavepacket maxima for $n=75$ and $n=32$ are evident from the plots of the magnitudes of the maxima that are plotted in figure $9(b)$. It can be seen that for early times, at least, the amplification experienced by the disturbance wavepacket, when measured in terms of the maxima of the $\omega_{\theta w}$, is not greatly different for $n=75$ and $n=32$.

In contrast, the time histories plotted in figure 10 provide further illustration of disturbance behaviour that appears to be more characteristic of an absolute instability. The azimuthal mode number is again taken to be $n=75$, but the disturbance is now 


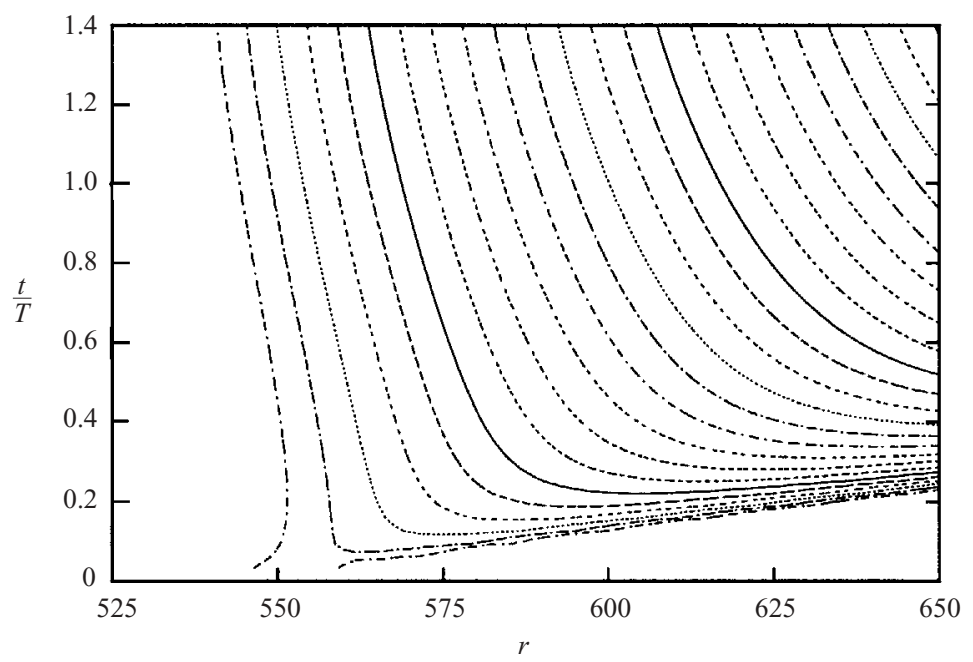

FIGURE 11. Spatio-temporal development of $\left|\omega_{\theta w}\right|$ for an impulsively excited disturbance with $n=75$. The impulsive disc surface motion used to excite the disturbance was centred at $r_{e}=550$. (Contours drawn using a logarithmic scale, with levels separated by factors of two.)

impulsively excited at $r_{e}=550$ (see Point 3 in figure $2 b$ ) instead of at $r_{e}=500$. The excitation now lies well inside the absolutely unstable region according to the SH linear stability theory of Lingwood (1995) - in fact, the situation corresponds to figure $3(b)$. It can be seen that there is temporal growth at all selected radial positions. In particular, the disturbance grows at $r=r_{e}=550$. It also grows at $r=525<r_{e}$. At this inward position the surface motion associated with the 'impulsive' forcing was relatively small, but not completely negligible. Nevertheless, we do not believe that the continual temporal growth observed at $r=525$ is simply the localized response to a locally prescribed wall motion of small but finite extent. Temporal growth was not sustained at $r=525$ for the previous case with $r_{e}=500$ (see figure 5), yet the distance from the centre of the excitation is the same, albeit in the opposite radial direction, and consequently the small wall motion associated with the forcing would have been very similar. These differences in the disturbance behaviour at $r=525$, between two simulations conducted with different forcing locations, show that there can be upstream information transmission in the radial direction. Stronger evidence for the existence of substantial radially inward propagation effects can be obtained by examining the overall development of the disturbance wavepacket for $n=75$ and $r_{e}=550$, using spatio-temporal contour plots, as before. From the contours displayed in figure 11 it is evident that, over the time interval considered, the trailing edge of the disturbance propagates along the inward radial direction, rather than radially outward as was the case when the excitation was applied at $r_{e}=500$. However, it may also be observed that the temporal growth experienced by the disturbance at any given radial position remains rather weak in comparison with the convective growth revealed by tracing the wavepacket amplitude maximum.

Figure 12 presents results from a simulation of disturbance evolution from an impulsively excited disturbance in the approximate SH flow. The azimuthal mode number was again taken to be $n=75$ and the Reynolds number set equal to $R=550$, in order to facilitate comparisons with the SI simulation described immediately above. A comparison of figures 11 and 12 makes it plain that the inward radial propagation is 


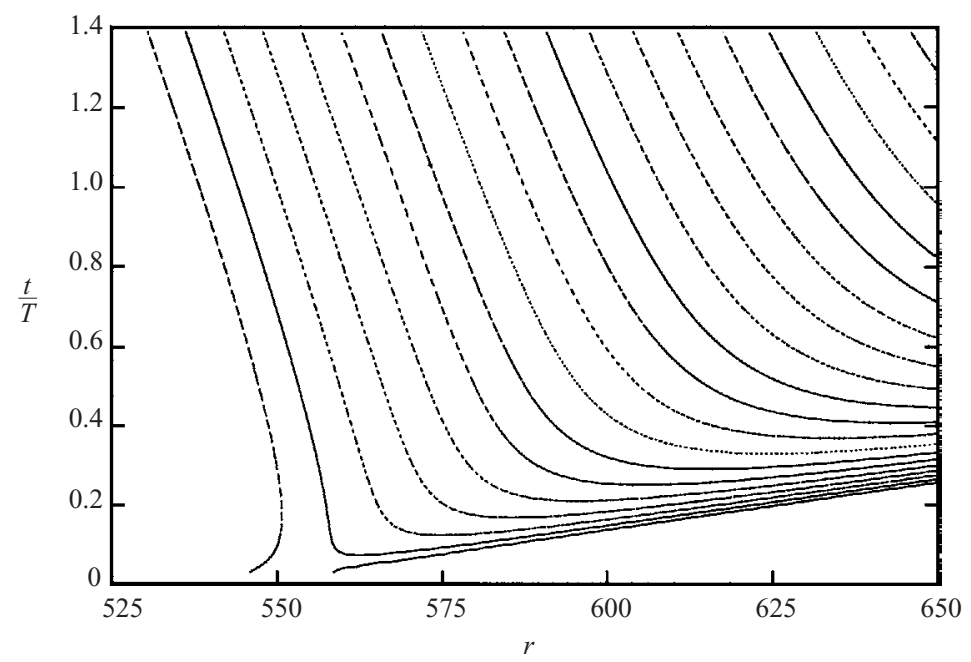

FIGURE 12. Spatio-temporal development of $\left|\omega_{\theta w}\right|$ an impulsively excited disturbance in a spatially homogeneous flow. The azimuthal mode number is $n=75$ and the Reynolds number is $R=550$. The arbitrary origin for the radial coordinate has been chosen so that the impulsive disc surface motion was centred at $r_{e}=550$. (Contours drawn using a logarithmic scale, with levels separated by factor of two.)

more pronounced in the $\mathrm{SH}$ case. This is as might be expected. For the SI simulation, the radially inward direction corresponds to decreasing Reynolds numbers and hence decreasing instability for the disturbance. When the SH approximation is applied, all radial locations become equivalent because they are all associated with the same Reynolds number. Consequently, the disturbance is not subject to any stabilization as it propagates radially inwards. The effects of the mean-flow SI on the evolution of the wavepacket amplitude maximum are also as might have been anticipated. In the real SI flow the radially outward propagation exhibited by the amplitude maximum is associated with an increasing local Reynolds number, and hence with greater instability. Thus the temporal growth of the wavepacket amplitude maximum is enhanced, as may be confirmed upon making reference, once more, to the detailed differences between the contours plotted in figure 11 and figure 12 .

Further comparisons between the data obtained from the SI and SH simulations reveals the existence of more subtle effects attributable to the mean-flow SI. Figure 13 contains a plot that illustrates the temporal evolution of the disturbance for the $\mathrm{SH}$ case with $R=550$. The figure also includes a replot of the evolution obtained at the corresponding radial position $r_{e}=550$ in the real SI boundary layer. It can be seen that the two plotted curves are quite close together for the early part of the time interval. However, over a longer time period, there is weaker accumulated growth in the SI case, together with a phase shift that is indicative of disparate temporal frequencies. Differences in the growth rates and the frequencies can be identified, in a more precise manner, by considering the complex-valued quantity defined by

$$
\tilde{\beta}=\frac{\mathrm{i}}{A} \frac{\partial A}{\partial t},
$$

where $A$ is some measure of the disturbance amplitude that is known for each radial position and time instant. In keeping with what was done before, we will choose $\omega_{\theta w}$ for $A$. Provided that $\tilde{\beta}$ is found, in practice, not to vary too rapidly in either 


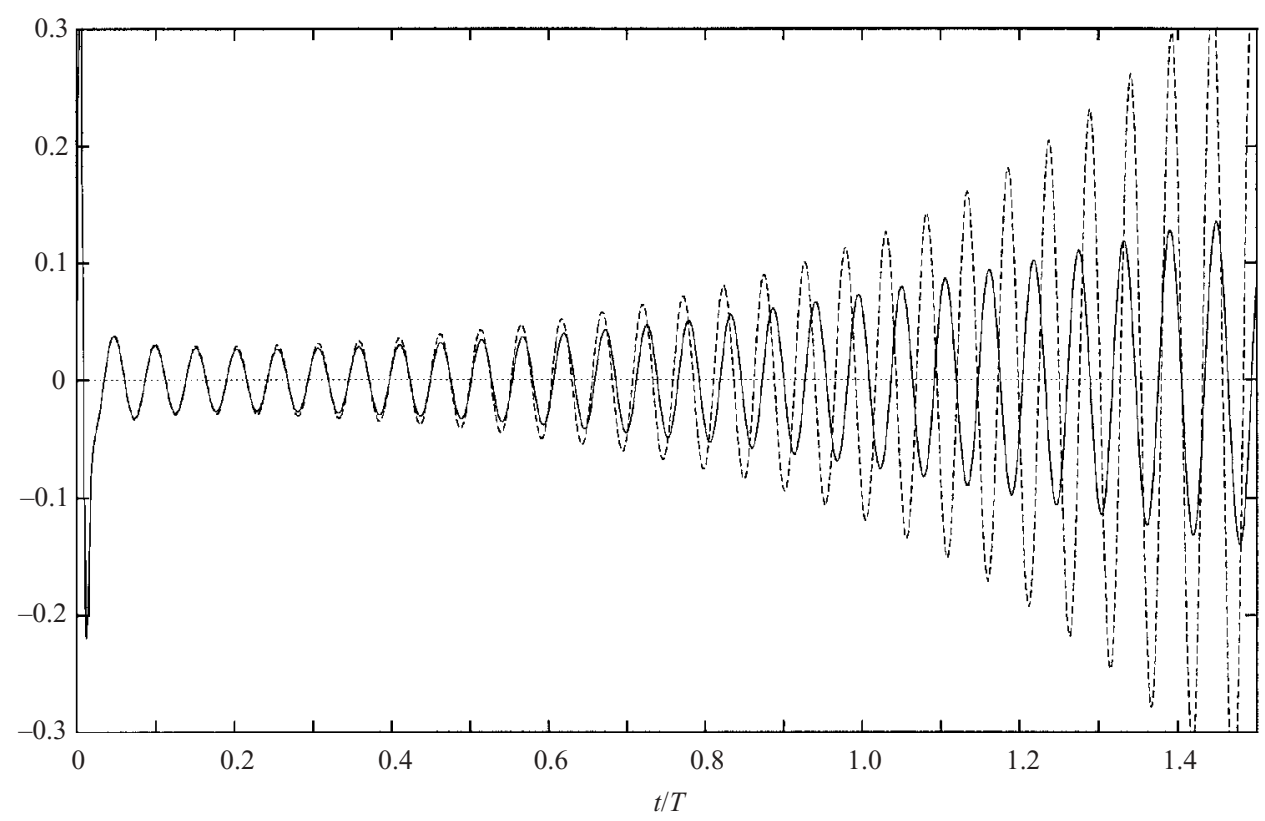

FIgURE 13. Comparison of the variation of $\omega_{\theta w}$ for a disturbance with $n=75$ evolving in spatially homogeneous and inhomogeneous flows. The temporal development is shown for the radius $r=r_{e}$ where the impulsive excitation was centred. Solid line: inhomogeneous flow with $r_{e}=550$; dashed line: homogeneous flow with $R=550$.

space or time, its real and imaginary parts may be interpreted as being, respectively, the local temporal frequency and the local temporal growth rate of the disturbance. This interpretation presumes that there is only a single mode of disturbance with a significant level of excitation at any specific radial location and time. The identification of the real and imaginary parts of $\tilde{\beta}$ as the unique local temporal frequency and growth rate would not be appropriate if there were a superposition of a number of discrete modes of disturbance, each with a different characteristic frequency.

Figure 14 displays the local temporal frequencies and growth rates, computed using $\omega_{\theta w}$, for the $\mathrm{SH}$ simulation. Plots are given for $r=r_{e}$ and for an additional three equally spaced positions that are taken radially inwards and outwards from $r=r_{e}$. It may be observed that there is a constant-frequency response at $r=r_{e}$. (Note that the evolution for the very earliest times has been deliberately omitted from the figure. This has been done to avoid difficulties of interpretation during the initial transient phase. Owing to the impulsive forcing many other modes of disturbance are initially excited with a wide range of frequencies. These other modes may initially obscure the single mode that eventually becomes dominant.) The temporal frequency of the disturbance at the other three radial positions can be seen to asymptote to the constant frequency found at $r=r_{e}$. It may be surmized that, as time passes, the response at every radial position settles upon a single frequency. This behaviour is not very surprising, since all radial positions are equivalent for the approximate $\mathrm{SH}$ flow. The equivalence between different radial positions is also evidenced by the plots for the locally defined temporal growth rates. It appears that, for all of the selected radial positions, the growth rates asymptote to a constant value. However, the asymptotic approach to a constant value, at any given radial location, is somewhat more protracted for the growth rates 

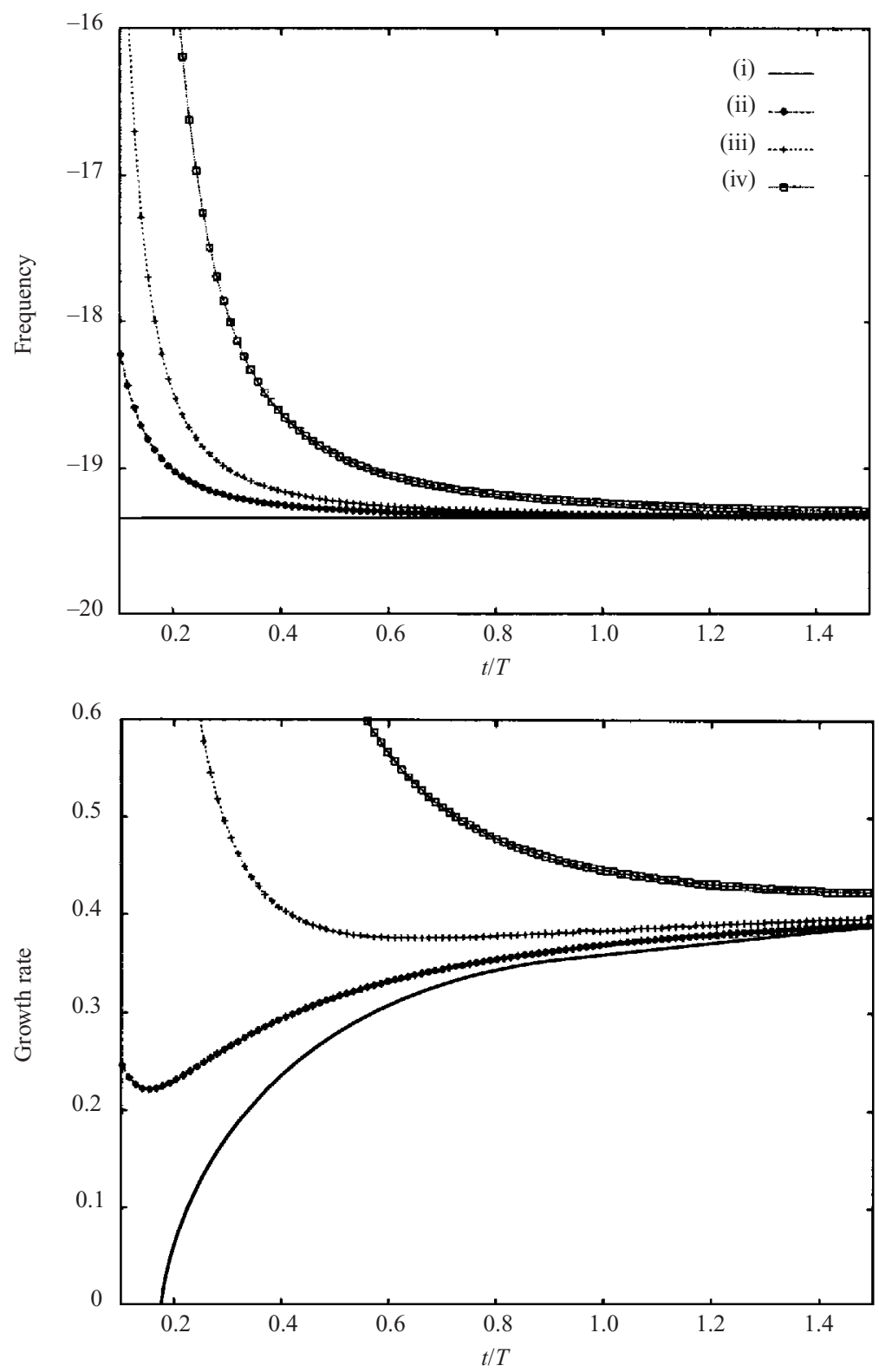

FiguRE 14. Local temporal frequencies $\tilde{\beta}_{r} R$ and temporal growth rates $\tilde{\beta}_{i} R$ for a disturbance with $n=75$ in a spatially homogeneous flow for $R=550$. The temporal development is plotted for four different radial positions: (i) $r=r_{e}$, (ii) $r=r_{e}-25$, (iii) $r=r_{e}+25$, (iv) $r=r_{e}+50$.

than for the frequencies. Thus for the SH flow, as expected from Lingwood's (1995) analysis, a response of the form (3) is generated.

Figure 15 displays local temporal frequencies and growth rates that were obtained from the SI simulation. The selected radial positions are the same as for figure 1. It is immediately obvious that in this case there is no tendency to asymptote to a constant frequency. The local frequencies evolve, initially, in the same manner as for the SH case. But after the initial period their magnitudes fall at all radial locations. 

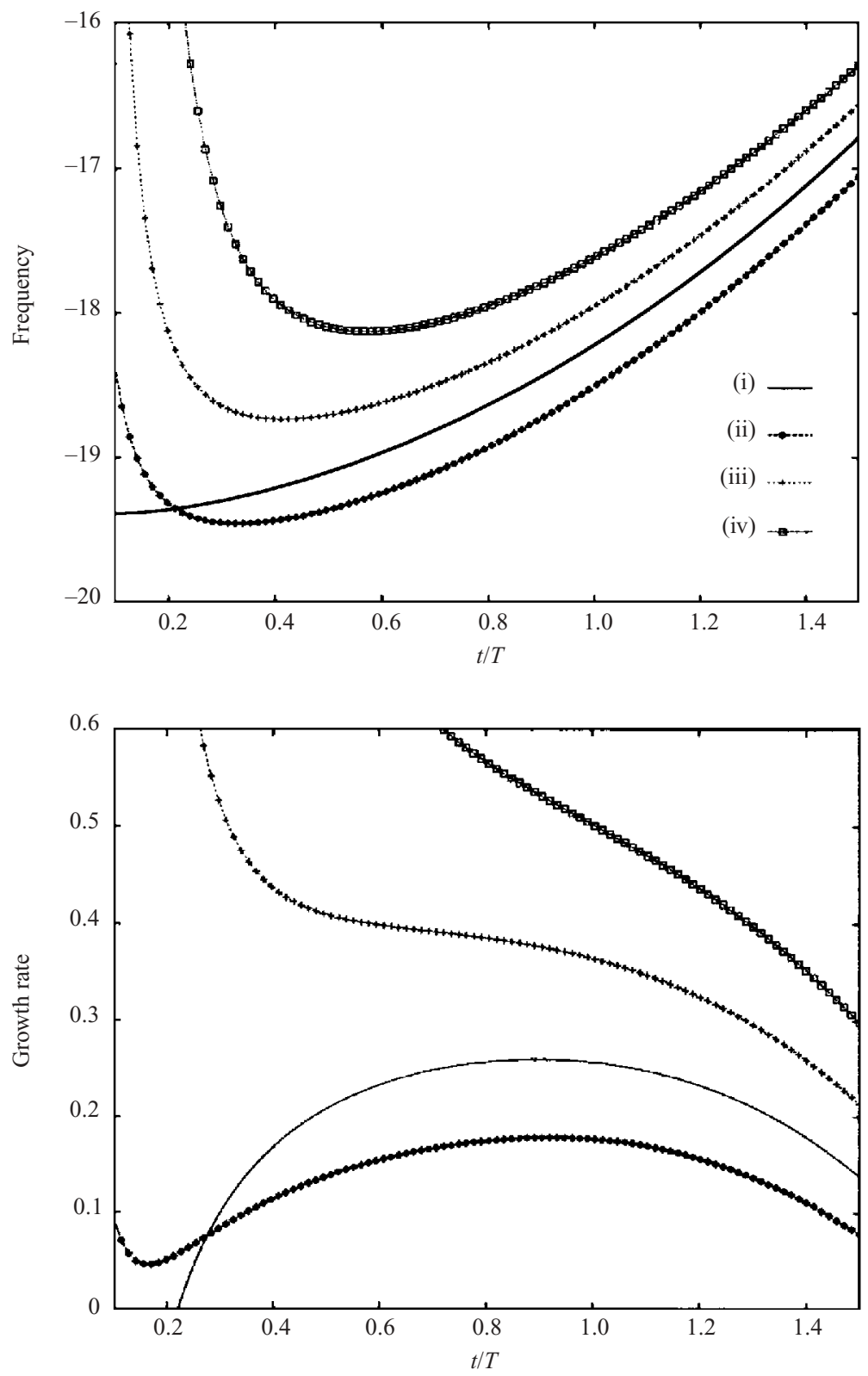

FiguRE 15. Local temporal frequencies $\tilde{\beta}_{r} R$ and temporal growth rates $\tilde{\beta}_{i} R$ for a disturbance with $n=75$ developing in a spatially inhomogeneous flow. The impulsive excitation was centred at $r_{e}=550$. The temporal development is plotted for four different radial positions: (i) $r=r_{e}=550$; (ii) $r=525$; (iii) $r=575$; (iv) $r=600$.

(Note that the frequencies are negative in figures 14 to 16.) The local temporal growth rates also exhibit significantly different behaviour compared with the SH flow. For all radial locations the long-term trend is for the growth rate to diminish steadily with time. It appears from this trend that the growth rates would eventually either asymptote to zero or become negative. Unfortunately, longer simulations, which could have confirmed whether or not the disturbances ultimately decayed, were extremely 
difficult to carry out. Convergence problems were encountered with the iteration scheme used to solve the discretized governing equations, owing to the very large range of disturbance magnitudes that developed within the computational domain. In the absence of any nonlinearity, the maximum amplitude of the disturbance wavepacket was free to exhibit unhindered exponential growth. (For $t / T \sim 1.4$ the maximum disturbance wavepacket amplitude was more than $O\left(10^{20}\right)$ times larger than the amplitude found at $r=r_{e}$. The incommensurability between the temporal growth at a fixed radial position and the growth obtained by following the trajectory of the wavepacket amplitude maximum was discussed above.)

These simulation results suggest that the global behaviour may well be convective. But numerical problems prevented us from continuing the simulations for a sufficiently long time to provide more definite evidence. To overcome these numerical problems we carried out another series of simulations for which $r_{e}=530$ and $n=67$. These parameters still lie well within the region of absolute instability - see Point 4 in figure $2(b)$ - but the absolute instability is less powerful than for the previous simulation. Figure 16 plots the variations in the temporal growth rates and frequencies corresponding to these new parameters for both SI and SH simulations. In this case the closer proximity to the boundary of the absolutely unstable region permits a more definite identification of the changeover from temporal growth to temporal decay. The locally defined growth rates and frequencies determined from the SI simulation are plotted for equally spaced radial positions, as before. For the SH simulation, the values for the temporal growth rate and frequency are only displayed for $r=r_{e}$, using the same axes as for the SI results. (The SH frequency agrees exactly with the absolute-instability frequency given by Lingwood (1995) for the same combination of parameters.) It is now clear that in the SI flow the growth rates eventually become negative for all monitored radial locations. Thus the disturbance shows a long-term decay after an initial period of growth. This is particularly marked for $r=r_{e}$ where little growth occurs at all; for the more outboard radial locations there is much stronger transient growth before any decay sets in.

The results displayed in figure 16 strongly suggest that the long-term global behaviour is consistent with convective instability, i.e. the global behaviour is similar to that found in spatially inhomogeneous systems that are everywhere at most convectively unstable. The convective nature of the long-term behaviour is even more clearly apparent in the contrasting ray diagrams presented in figures $17(a, b)$. The $\mathrm{SH}$ simulation gives a pattern typical of absolute instability. For the SI simulation, on the other hand, the rays which initially propagate upstream, turn back on themselves in the manner suggested by figure 4(b). Although there is always room for doubt in any numerical simulation or physical experiment, the results displayed in figures 16 and 17 unmistakably indicate that the long-term global behaviour is not consistent with a linear amplified global mode of the form (3), i.e.

$$
A \sim \mathrm{e}^{-\mathrm{i} \omega_{G} t},
$$

but is, instead, consistent with convective instability. This conclusion cannot be dismissed as being due to a minor adjustment in the boundary of the absoluteunstable region caused by SI. Numerical simulations conducted for both the SI and SH flows for different azimuthal mode numbers, and also for an impulsive excitation applied at much higher Reynolds numbers, provided firm evidence that the convective-like long-term behaviour is, in fact, typical.

For instance a SI flow simulation conducted for $n=75$ and $r_{e}=650$ (see Point 5 of figure $2 b$ ) gave local temporal growth rates that showed a very clear long-term 

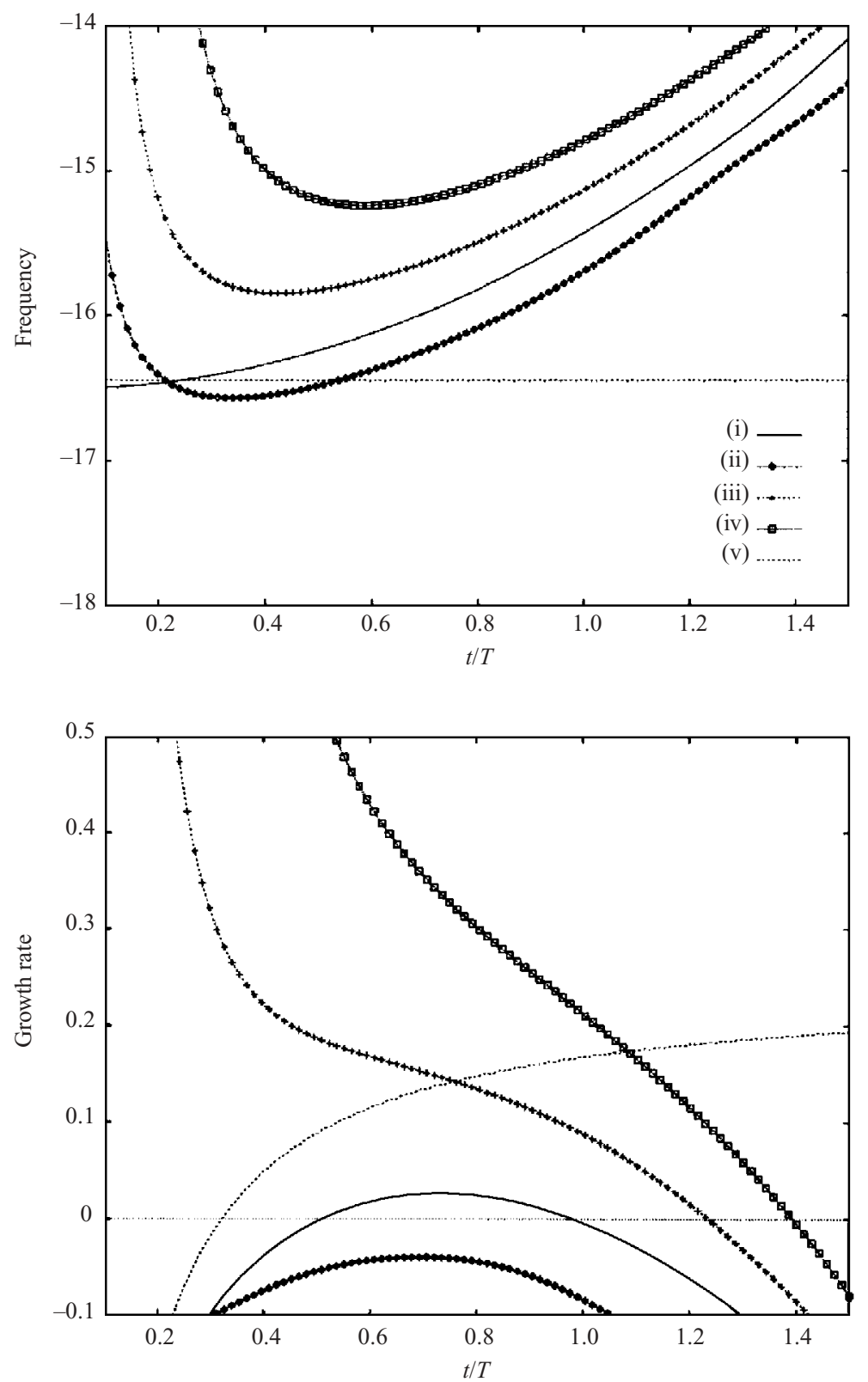

FIGURE 16. Local temporal frequencies $\tilde{\beta}_{r} R$ and temporal growth rates $\tilde{\beta}_{i} R$ for a disturbance with $n=67$. For the curves labelled (i)-(iv) the impulsive excitation was centred at $r_{e}=530$ and the flow is spatially inhomogenous. The temporal development is plotted for the radial positions: (i) $r=r_{e}=530$; (ii) $r=505$; (iii) $r=555$; (iv) $r=580$. The curves labelled (v) show the development at the point of impulsive excitation of a similar disturbance in a spatially homogeneous flow with $R=530$. The constant frequency obtained from the simulation for the homogeneous flow is in exact agreement with the frequency of the absolute instability predicted by Lingwood (1995).

diminution, when compared with the corresponding $\mathrm{SH}$ simulation. The local growth rates, computed for a range of radii around the impulse location, are plotted in figure 18, along with the corresponding temporal frequencies. It can be seen that 

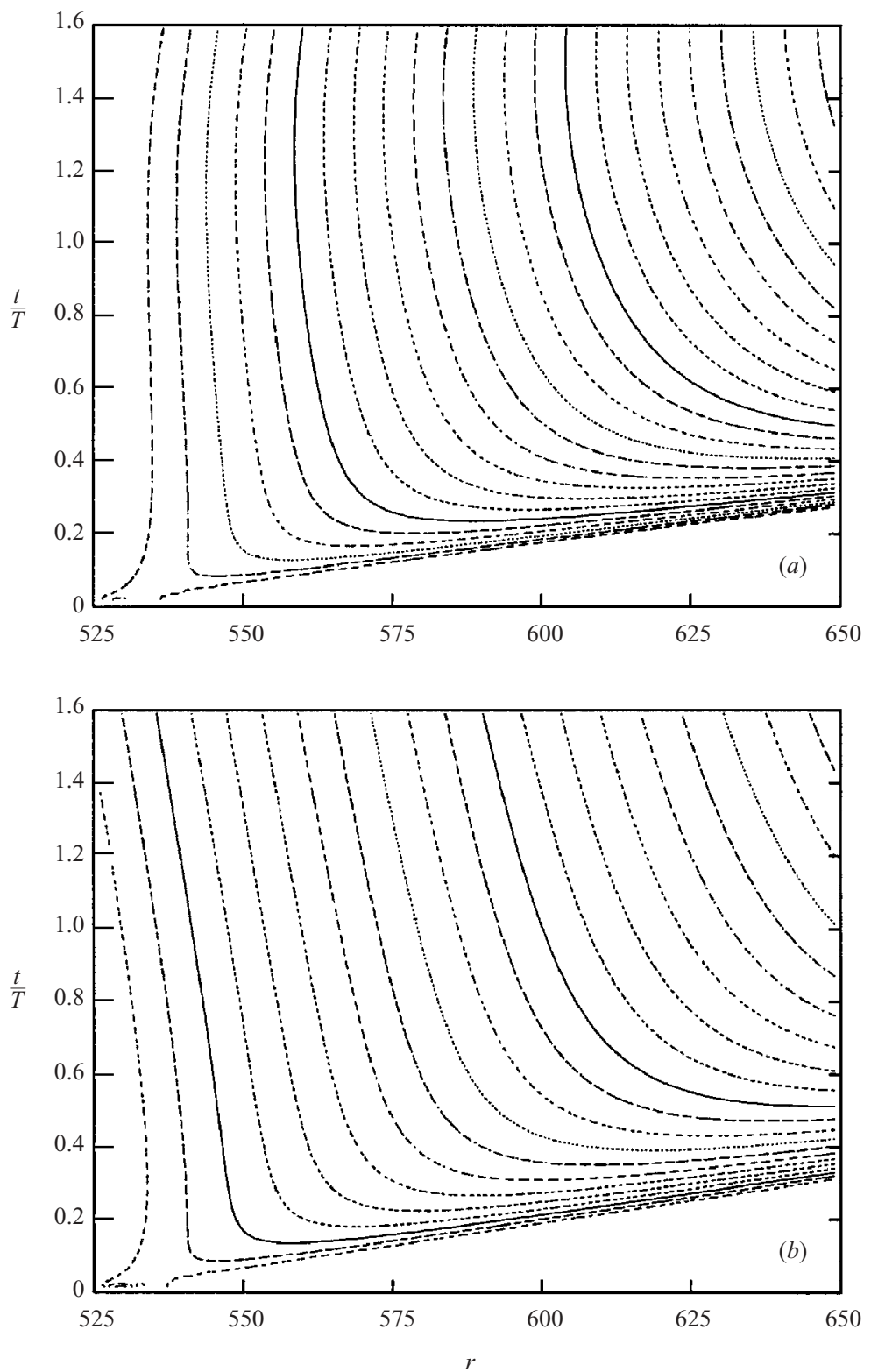

FiguRE 17. Spatio-temporal development of $\left|\omega_{\theta w}\right|$ for an impulsively excited disturbance with $n=67$. The impulsive disc surface motion used to excite the disturbance was centred at $r_{e}=530$. (a) Spatially inhomogeneous flow; $(b)$ spatially homogeneous flow with $R=530$. (Contours drawn using a logarithmic scale with levels separated by a factor of two.)

for the simulation with $n=75$ and $r_{e}=650$, the locally computed temporal growth rates show the same clear trend, namely to decay, as was previously identified for a simulation with $n=75$ and $r_{e}=550$. The qualitative form of the temporal behaviour is retained, despite the fact that increasing the Reynolds number from $R=550$ to $R=650$ produces more than double the temporal growth rate for the $\mathrm{SH}$ flow with $n=75$. Exactly the same kind of long-term behaviour was found in another example simulation that was conducted with $n=67$ and $r_{e}=750$ (see Point 6 of figure $2 b$ ). 

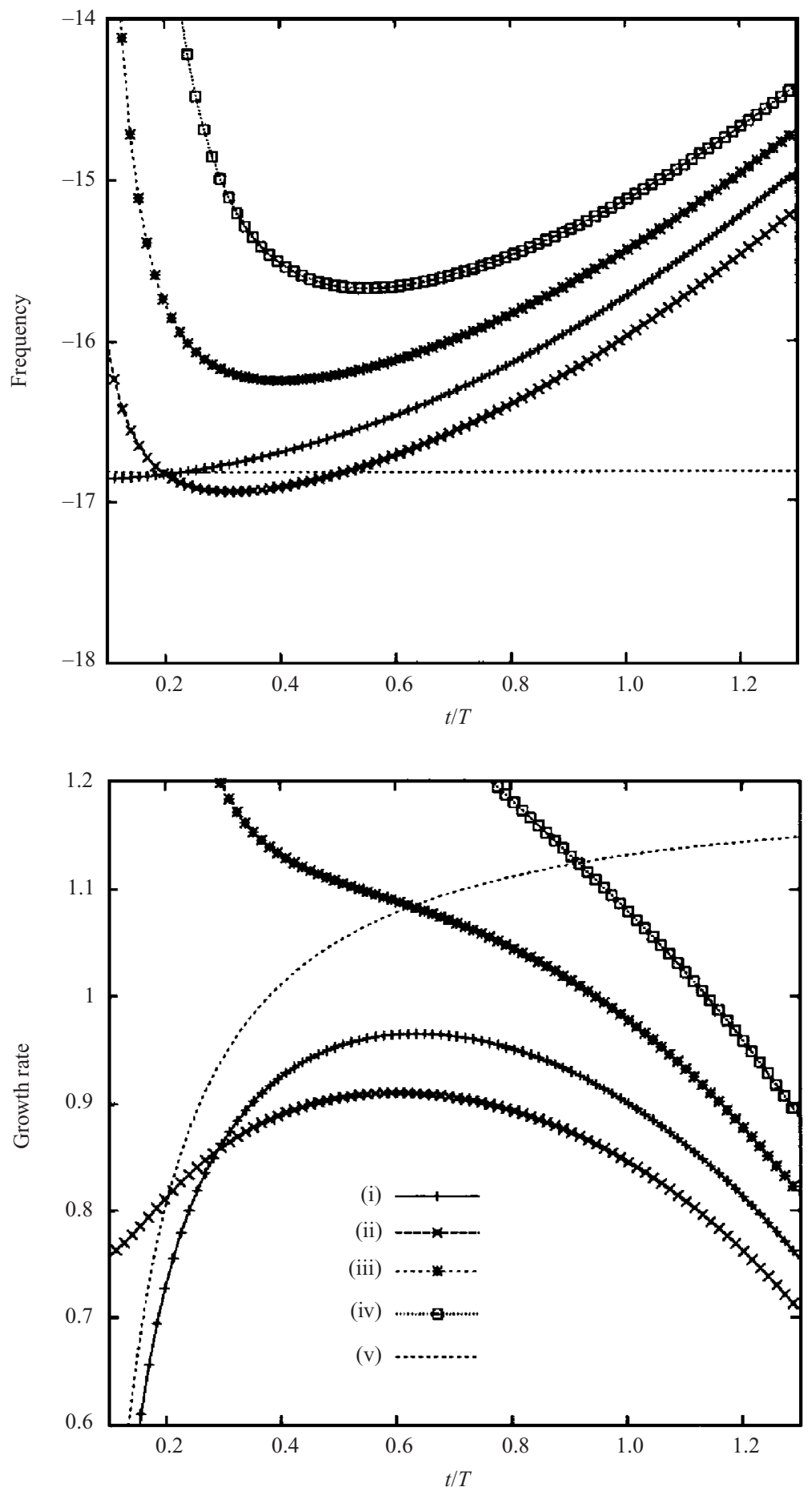

FiguRE 18. Local temporal frequencies $\bar{\beta}_{r} R$ and temporal growth rates $\bar{\beta}_{i} R$ for a disturbance with $n=75$. For the curves labelled (i)-(iv) the impulsive excitation was centred at $r_{e}=650$ and the flow is spatially inhomogeneous. The temporal development is plotted for the radial positions: (i) $r=r_{e}=650$; (ii) $r=625$; (iii) $r=675$; (iv) $r=700$. The curves labelled (v) show the development at the point of impulsive excitation of a similar disturbance in a spatially homogeneous flow with $R=650$. 


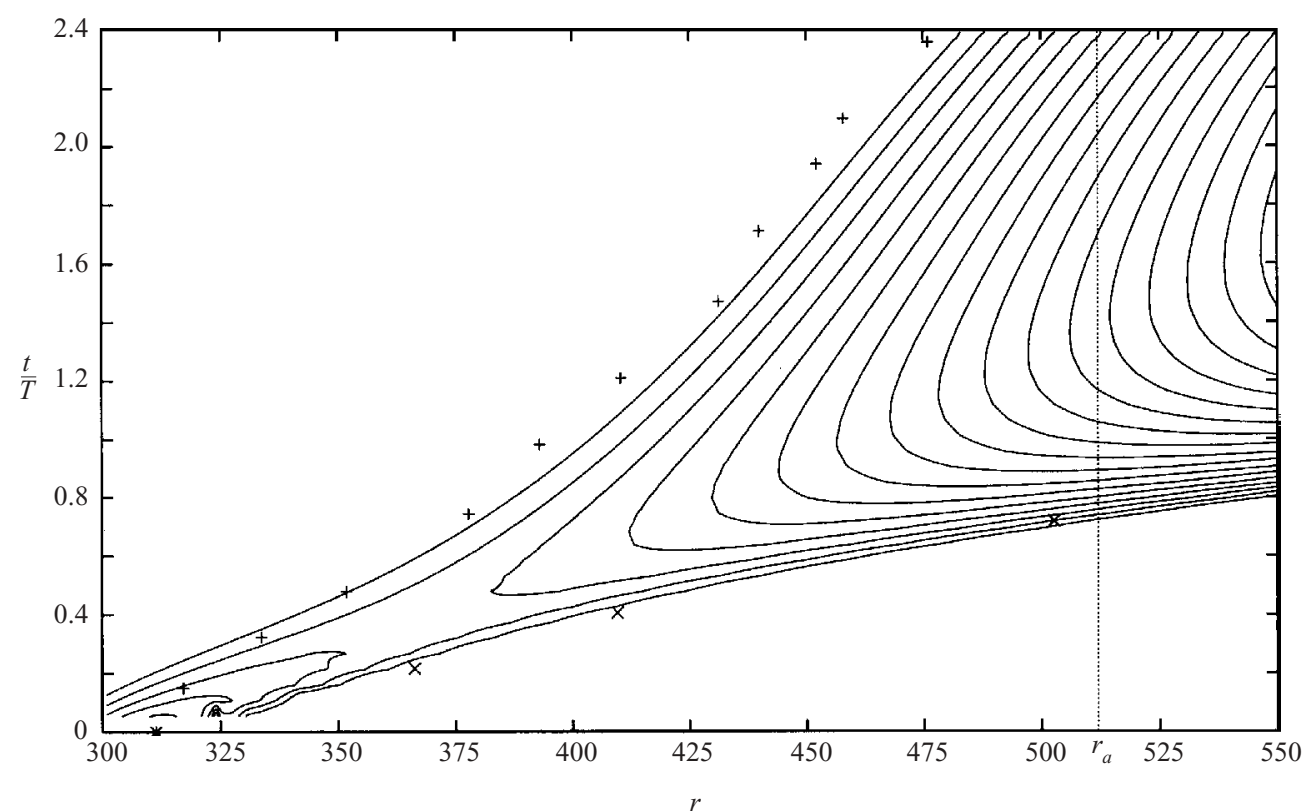

FiguRE 19. Spatio-temporal development of $\left|\omega_{\theta w}\right|$ for an impulsively excited disturbance with $n=67$ and $r_{e}=311 . \times,+$ denote experimental data points for the leading and trailing edges of the wavepacket taken from figure 15(b) of Lingwood (1996). (Contours drawn using a logarithmic scale with levels separated by a factor of two.)

In this case the disturbance was excited at a location even further into the region of absolute instability, at a radius that corresponds to approximately one and a half times the critical Reynolds number for the onset of absolute instability in the $\mathrm{SH}$ flow. This is very far beyond any radius at which the rotating-disc boundary layer has been found to remain laminar in physical experiments.

\section{Comparison with Lingwood's experimental data}

It is not possible to carry out a simulation that exactly mimics the experiment described in Lingwood (1996). Both the numerical and physical experiments generate the initial disturbances with excitations that roughly approximate a point impulse. Thus a wide range of frequencies and azimuthal wavenumbers are generated in each case. Lingwood excited the disturbances by a pressure pulse generated by a small transient jet that was triggered once per revolution of the disc. The precise spectral content of the initial experimental disturbances is not known, but it is likely to differ considerably from that of the numerically generated disturbances. Thus we cannot sensibly compare experimental and numerical wavepackets containing many different azimuthal wave-numbers because we have no way of knowing the relative amplitudes of the azimuthal modes in the experiment. All we can sensibly do is compare the numerically simulated development of an impulsively excited wavepacket containing a single azimuthal mode with that of the experimentally generated wavepacket. The logical choice for the single azimuthal mode is $n=67$, as this is the first to become absolutely unstable.

Figure 19 displays space-time contours obtained from a SI numerical simulation of a disturbance with $n=67$, that was excited by an impulse centred at $r_{e}=311$ like 
the experiment. Now the impulse is located well inboard of the region of absolute instability - see Point 7 in figure 2(b). This situation corresponds to figures $3(a)$ and 4(a) and to Lingwood's (1996) experimental investigation. Lingwood applied a pressure impulse through a hole in the rotating disc surface and then tracked the evolution of the leading and trailing edges of the resulting wavepackets. It can be seen that reasonably good agreement is found in figure 19 between the leading and trailing edges of the numerically simulated wavepacket and Lingwood's experimental data. She argued that her experimental data for the trailing edge of the wavepackets could be fitted well by a curve that asymptoted to a constant radius as $t \rightarrow \infty$. The constant radius selected corresponded, of course, to the critical value for absolute instability.

It is only fair to point out that the choice of threshold disturbance amplitude marking the leading and trailing edges of the numerically generated wavepacket in figure 19 is somewhat arbitrary. The choice of threshold amplitude makes little difference to the location of the leading edge, but the precise location of the trailing edge is quite sensitive to the threshold level chosen. In contrast, the slope of the trailing edge is insensitive both to the precise value of $n$ as long as it is fairly close to $n=67$, and to the value of the threshold amplitude. Furthermore, only a single azimuthal mode is included in the numerical simulation, whereas the experimentally generated wavepacket contains a superposition of azimuthal modes. We have carried out similar numerical simulations for a range of azimuthal modes corresponding to values of $n$ between 30 and 75. The azimuthal mode with $n=67$ is the first to become absolutely unstable. This is why it was chosen for figure 19. For $r>r_{a}$ the value of $n$ corresponding to the most unstable azimuthal mode rises. As $n$ rises up to $n=75$, the slope of the wavepacket's trailing edge tends to become steeper. The leading edge is insensitive to the value of $n$ or $r$. It is plain from figure 19 that the $n=67$ mode is initially damped for $r>r_{e}$. What probably happens with the experimental wavepacket is that at relatively low values of $r>r_{e}$, it is initially dominated by the most convectively unstable modes $(n \sim 30)$. But absolutely unstable modes dominate the wavepacket at later times as $r \rightarrow r_{a}$.

Thus we can conclude from figure 19 that the simulations are consistent with the experiments. Furthermore, the numerical simulation does not appear to be inconsistent with asymptotic behaviour proposed by Lingwood. It is plausible, on a first inspection, to believe that the disturbance trailing edge depicted in figure 19 is beginning to asymptote to a constant radius, rather than propagating further and further away from its source at $r_{e}=311$. Unfortunately it was not possible with Lingwood's experiments to follow the development of the wavepacket for times beyond about $t / T=2.6$ and to higher values of $r$ in order to establish more certainly that the trailing edge really does asymptote to a constant radius. Equally we cannot continue the numerical simulation for times beyond about $t / T=2.4$ owing, as explained above, to the enormous exponential growth of the convectively unstable component of the wavepacket. However, one advantage of the numerical, as compared with a physical, experiment, is that it is possible to monitor the development of the disturbances within the absolutely unstable region itself. Even over the limited time period of the present simulation, a more careful examination of the disturbance time histories at particular radial locations in the absolutely unstable region provides strong evidence against the existence of a temporally growing global mode. Figure 20 shows the time history for the $n=67$ disturbance development at $r=551$. As has been noted before, this location is well within the region of absolute instability. It can been seen that, once the disturbance has reached this location after propagating radially outwards 


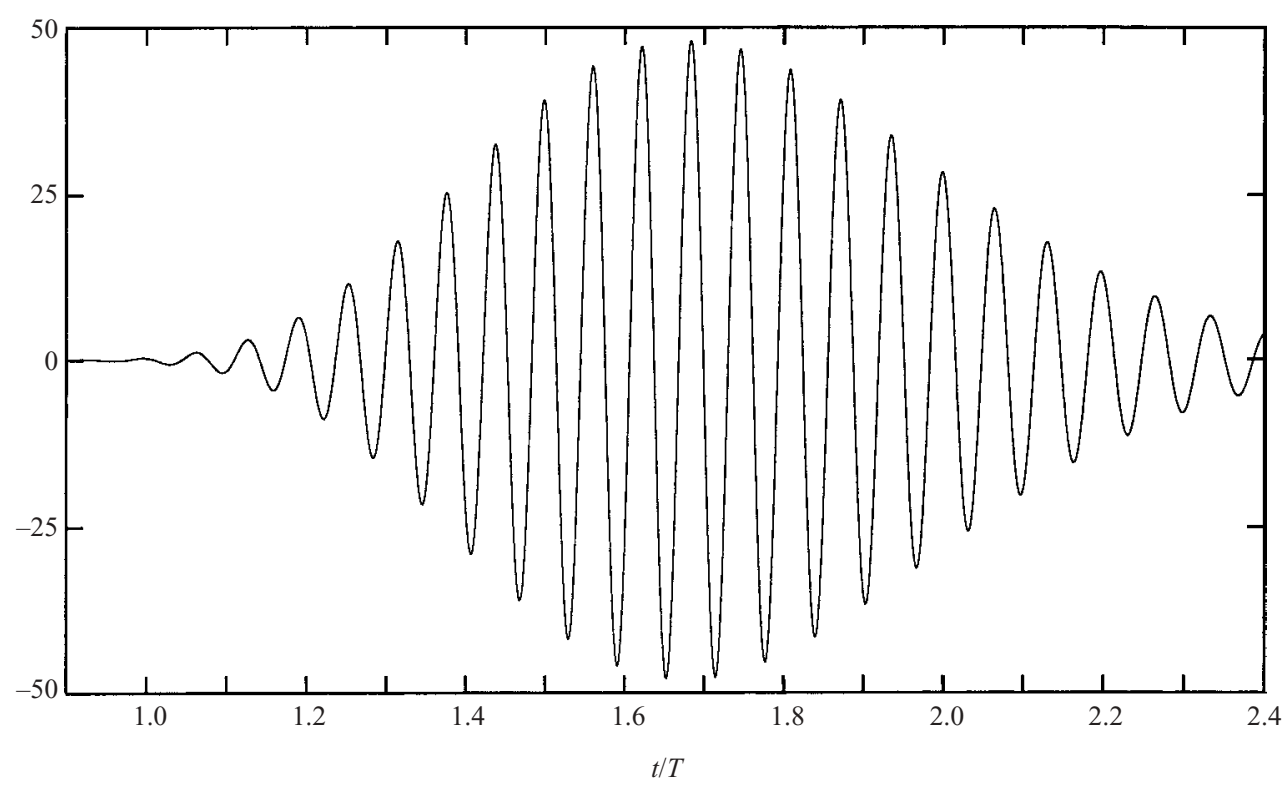

FIGURE 20. Variation of $\omega_{\theta w}$ for a disturbance with $n=67$ excited by an impulse centred at $r_{e}=311$. The temporal development is shown for $r=551$.

from its source, there is a period of rapid temporal growth which is followed by equally strong decay.

It could be argued that such behaviour is, perhaps, not necessarily inconsistent with the existence of a temporally amplified global mode. It is possible that such a local temporal maximum in the disturbance amplitude could come about because convective components of the disturbance, with frequencies different from those predicted for the absolute instability, are the first to arrive at the selected radial position. Thus the failure to observe any sustained temporal growth might be attributed to the limited time duration of the numerical simulation. Over a longer time interval, if a temporally growing global mode did exist, the disturbance would be expected to grow once more. An explanation along these lines might also account for the differences between the behaviour displayed in figure 17 and that depicted earlier in figure 11. As we have already suggested, it is conceivable that in the latter case the radially outward propagation of different frequency components excited by the impulse could lead to transient disturbance behaviour that would mask the appearance of any sustained temporal growth. However, an investigation of locally computed temporal frequencies strongly suggests that this explanation is unlikely to be correct. Figure 21 displays the local temporal frequencies at $r=551$ for $n=67$ and $r_{e}=311$. A line corresponding to the asymptotic frequency obtained from the SH flow simulation with $n=67$ and $R=551$ is also included. By making reference to the time history plotted in figure 21 it may be seen that the local temporal frequency defined at $r=551$ is close to that obtained from the SH simulation at exactly those times when the disturbance amplitude is approaching its maximum. In other words, the maximum amplitude occurs in the SI boundary layer when the disturbance is oscillating with a temporal frequency that corresponds to the absolute instability predicted for the $\mathrm{SH}$ flow. Such behaviour would not seem to be consistent in any obvious fashion with the existence of global mode having the form $A \sim \exp \left(-\mathrm{i} \omega_{G} t\right)$ in the SI flow. 


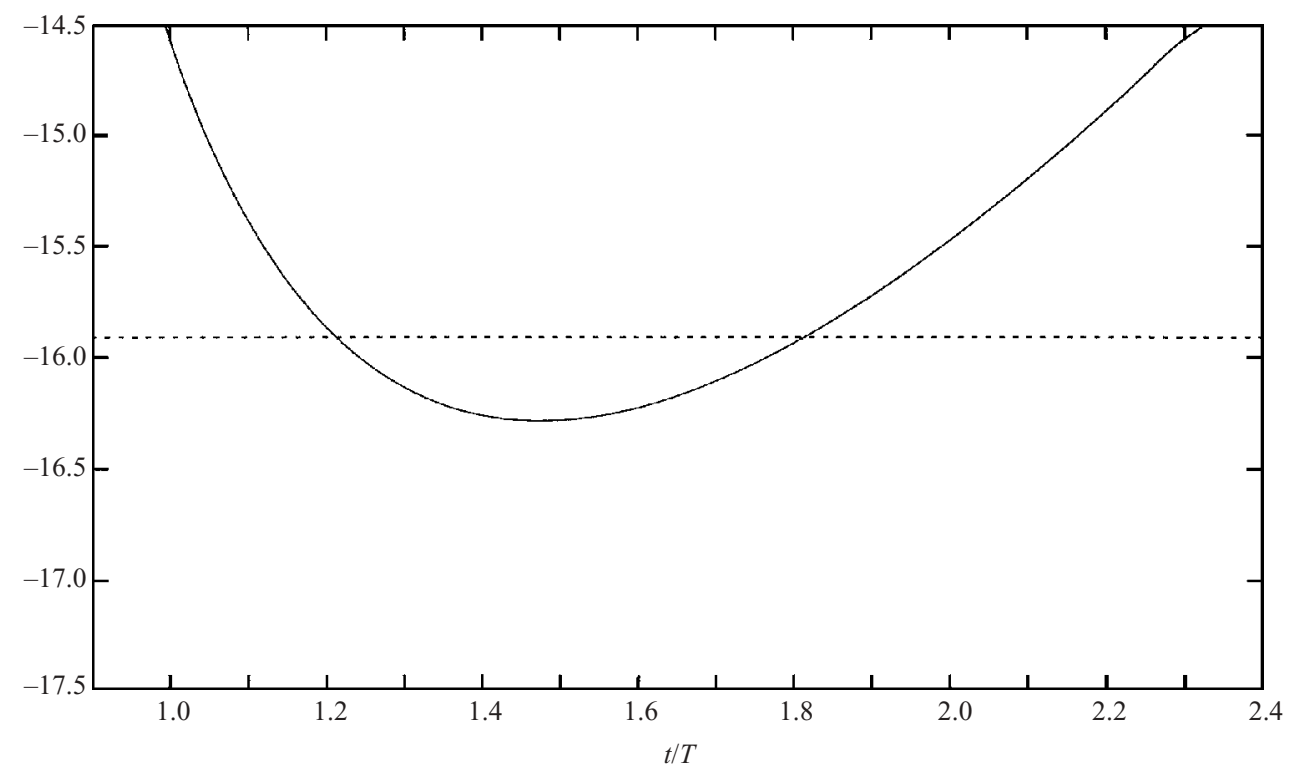

FIGURE 21. Variation of local temporal frequency $\tilde{\beta}_{r} R$ for a disturbance developing in the spatially inhomogeneous flow with $n=67$ excited by an impulse centred at $r_{e}=311$. The solid line plots the temporal development for $r=551$. The dashed line corresponds to the asymptotic frequency for the corresponding spatially homogeneous flow with $R=551$.

\section{Discussion}

The outcome of our investigation of the global behaviour of the rotating-disc flow has certainly surprised us. To some extent it runs counter to conventional wisdom. Accordingly, we would expect our numerical methodology to be questioned and will now discuss some of the possible numerical pitfalls. It is well known that the inflow and outflow boundary conditions can lead to spurious effects, even when carefully implemented. For example, Buell \& Huerre (1988) (cited in Cossu \& Loiseleux 1998) showed that spurious, destabilizing, pressure-feedback loops can be generated by the influence of the numerical outflow boundary conditions on an upstream boundary, causing flows that are everywhere convectively unstable to exhibit spurious selfsustained oscillations in the numerical simulations. Kaiktsis, Karniadakis \& Orszag (1996) also warn of the dangers of obtaining spurious results due to problems with the outflow boundary conditions when using numerical simulations to study global temporal modes. In their simulations of flow over a backward-facing step, they found that unless the computational domain was sufficiently long, the results were seriously affected when the disturbance was merely set equal to zero at the outflow boundary.

We were well aware of these problems and took great care, as described in I and $\S 2$, with our implementation of the outflow boundary conditions. Our computational domains extended very much further than the range shown in any of the figures displaying simulation results. Furthermore we ran extensive validation tests, some of which are reported in I. In the course of carrying out these tests we have carefully checked that the results presented here are independent of the size of the computational domain, providing it is sufficiently large. In any case, since our results are surprising because a sustained, temporally growing, global mode is not found, it is not likely that our simulations were adversely affected by the pressurefeedback problem identified by Buell \& Huerre. In this connection, it is worth noting 
again (see §2) that, in our experience also, spurious temporal growth resulted when computational domains were so short that unwanted feedback occurred from the outflow boundary.

A different numerical problem has been recently revealed by Cossu \& Loiseleux (1998). They have investigated the numerical dispersion relations obtained for the linear Ginzburg-Landau equation (see §1) using the Euler explicit and implicit, and Crank-Nicholson schemes. For example, in the case of the last of these three schemes, they found that in cases where an absolute instability should exist, there is a range of spatial step sizes for which it is impossible to obtain an absolute instability numerically, irrespective of the time step. This problem only existed for relatively small values of $\mu$ in (4). Nevertheless, it provides a valuable warning that numerically stable schemes may still exhibit spurious convectively unstable behaviour, or for that matter erroneous absolutely unstable behaviour. For our simulations, we used schemes with better stability characteristics than Crank-Nicholson (see I). But, probably, the best evidence that we were not unwittingly suffering from the problems analysed by Cossu \& Loiseleux is the fact that our numerical simulations of the (artificial) spatially homogeneous flow always correctly identified the type of instability (i.e. whether absolute or convective), appropriate to the choice of flow parameters, in accordance with Lingwood's (1995) stability theory. One final point may be worth making à propos the reliability of the computational results. This concerns our use in I of an inertial frame fixed with respect to the rotating disc for the formulation. Recently we have reformulated the governing equations and numerical schemes in terms of a fixed laboratory coordinate system. The results thus obtained were indistiguishable from those presented here.

The picture emerging from our simulations is summarized schematically in figures $4(a)$ and $4(b)$. The long-term global behaviour appears to be convective in the real spatially inhomogeneous flow. The absolute instability does not lead to sustained temporal growth. However, there is strong, localized, temporal growth, accompanied by upstream (inboard) propagation, over relatively short time scales. In this respect the behaviour is not unlike localized algebraic growth. This localized growth would ensure that the already existing, strongly growing, convective disturbances grow very strongly in the vicinity of the absolute instability. This localized growth is likely to be more than sufficient to bring in the nonlinear effects that govern the final stages of transition. The associated short-term upstream propagation would also ensure that the transition point was well-defined and not sensitive to background noise. Thus the picture revealed by the simulations and depicted in figures $4(a)$ and $4(b)$ is fully consistent with Lingwood's (1996) experiments.

Given the discussion in the previous paragraph, would the existence or otherwise of a linear, temporally growing, global mode have any implications for the observable physical behaviour of the flow? Or, is it merely a question of mathematical interest? On one level it certainly has important physical implications. Had such a global mode existed, we would have expected that the flow downstream of the onset of absolute instability would have behaved like a self-sustained oscillator. In practice, this would mean that the spectrum of the turbulent boundary layer would have been dominated by the global frequency, much like the turbulent wake behind a circular cylinder. Assuming that the picture revealed by our simulations is correct, what we would expect to see is a boundary layer dominated by convective structures. The three-dimensional, turbulent boundary layer on the rotating disc is unlikely to be closely similar to that over a flat plate; but, at least, without the global mode, it should not be radically different on a qualitative level. 


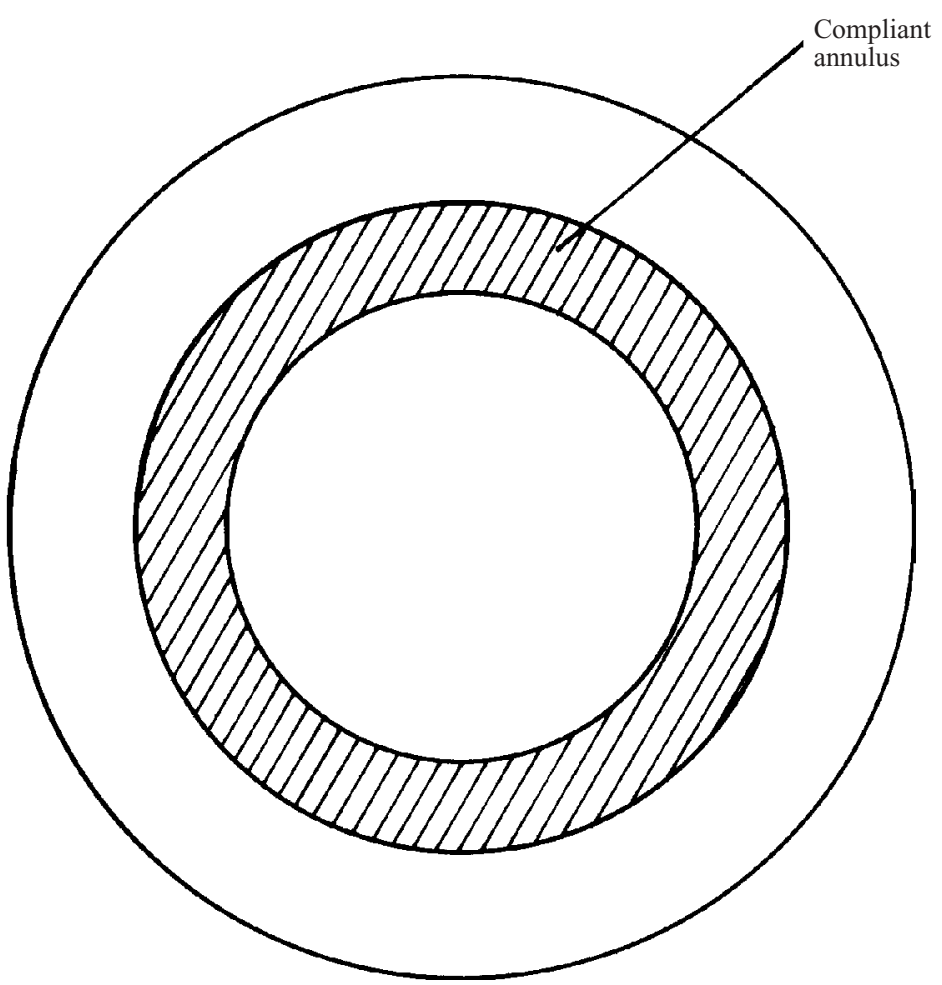

FIGURE 22. Schematic sketch showing a compliant annulus set into the disc surface.

\section{Numerical simulations for compliant rotating discs}

There is a continuing interest in the use of wall compliance as a method of laminarflow control (see Carpenter, Davies \& Lucey 2000, 2001 for recent reviews). In recent years there have been theoretical (Cooper \& Carpenter 1997a,b), experimental (Colley et al. 1999), and numerical-simulation studies (see I) of the effects of wall compliance on boundary-layer stability and transition over the rotating disc. In particular, Cooper \& Carpenter (1997b) demonstrated theoretically that only a small degree of wall compliance is sufficient to postpone the onset of absolute instability to much higher Reynolds numbers or suppress it entirely. It is therefore of interest to examine briefly the effects of wall compliance in the present numerical-simulation study. There is further theoretical motivation for such a study arising from the possibility of using wall compliance to reduce the extent of the absolutely unstable region. Had we found that the unbounded absolutely unstable region (recall that for fixed $\beta$ it is finite, albeit extensive, in extent) existing over purely rigid rotating discs was associated with a globally amplified mode as described in Huerre \& Monkewitz (1990), it would have been interesting to see how large the absolutely unstable region needed to be for the existence of global instability. But our study strongly suggests that there is no long-term temporal growth anywhere on the disc. Instead the absolute instability is associated with strong transient temporal growth. Accordingly, we might ask whether reducing the extent of the absolutely unstable region suppresses even this transient temporal growth, just as in cases where it exists the global instability can be suppressed if the absolutely unstable region is too small. 
The formulation and numerical methods described in I allowed us to make part of the disc's surface compliant. In fact, results were presented in I showing the evolution of disturbances generated by periodic wall forcing in the boundary layer over a disc with a compliant annulus, as shown schematically in figure 22 . It was shown that the Type I disturbances were subject to significant stabilization. The stabilizing effect on the Type II disturbances was found to be much weaker, with negligible modification to radial growth rates. In both cases the surface stiffness parameters that characterize the spring-backed plate model used for the compliant part of the disc were chosen so as to avoid the onset of flow-induced surface instabilities. It was noted that our numerical simulation results in I were in broad agreement with the linear stability results obtained by Cooper \& Carpenter (1997a) for a compliant surface formed from a single visco-elastic layer.

We will now present the results obtained from a numerical simulation of the development of a disturbance generated by an impulsive excitation of the real spatially inhomogeneous flow when part of the rotating-disc surface incorporates a compliant annulus. As in one of the numerical simulations described before, the azimuthal wavenumber of the disturbance was $n=75$ and the prescribed impulse centred around $r_{e}=550$. The surface was compliant over an annular region ranging from $r=600$ to 650. (The inner radial boundary of the compliant annulus thus lies well beyond the experimentally observed transition point. It also lies well within the absolutely unstable region determined by Lingwood's linear stability analysis. Similar, but less clear, effects were obtained when the inner boundary of the compliant annulus was located at a smaller radius.) The non-dimensional surface compliance parameters were selected in accordance with the optimization scheme described by Carpenter $\&$ Morris (1990). The flow-induced surface modes were expected to be, to a first approximation, marginally stable at the outermost radius of the compliant insert. The non-dimensional critical wavenumber, $\bar{\alpha}_{d}$, for the divergence mode, in terms of which the optimized compliant surface parameters could all be expressed, was chosen to have the value 0.2 .

The presence of the compliant annulus eliminates the absolute instability for $r \geqslant 600$. Of course, owing to the finite width of the annulus, technically the absolute instability reappears for $r \geqslant 650$. Our interest lies in the disturbance evolution in the region $r \leqslant 650$. The numerical simulations show that restricting the size of the region of absolute instability greatly weakens the transient temporal global amplification. This is clear from the simulation results presented in figure 23. Here the temporal development of the disturbance is plotted at several radial positions spaced at equal intervals outwards from $r=r_{e}$. Corresponding disturbance amplitude envelopes are also plotted for the case where the disc surface is entirely rigid. It is clear that the compliant annulus exerts a considerable stabilizing influence. Although the disturbance grows over a short time interval in all cases, the medium-term temporal growth of the disturbance is reversed, or very substantially diminished, at each of the radial positions considered. It is particularly interesting to note that the disturbance is stabilized at the two radial positions inboard from the inner boundary of the compliant annulus. The compliant wall has a strong influence inboard (i.e. upstream) on the flow over the rigid wall. In fact, as time progresses the influence of the compliant part of the surface becomes more extensive over the inboard (i.e. upstream) region. It is worth observing, however, that such behaviour is highly elliptic and could not be simulated by numerical methods that rely on the perturbations evolving in a quasi-parabolic manner. 

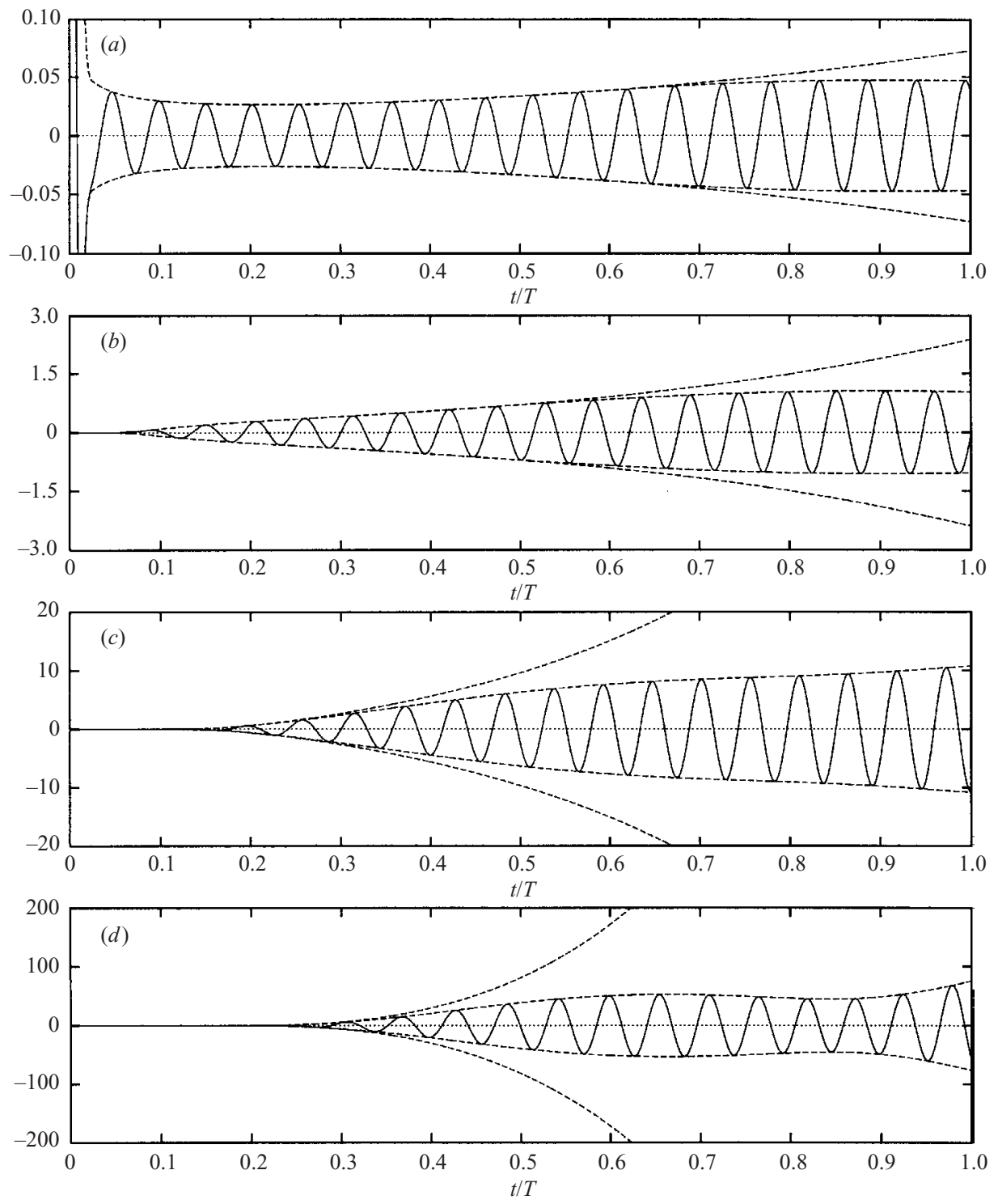

FIGURE 23. Time histories of $\omega_{\theta w}$ (solid lines) for a disturbance with azimuthal mode number $n=75$ that is impulsively excited at $r_{e}=550$. The disc surface is compliant for $600 \leqslant r \leqslant 650$. Amplitude envelopes $\pm\left|\omega_{\theta w}\right|$ are also shown (broken lines), both for the simulation with the compliant insert and for a corresponding simulation where the disc surface was entirely rigid. (a) $r=r_{e}=550 ;(b) r=575 ;(c) r=600 ;(d) r=625$. The surface compliance parameters are such that, notionally, there is marginal stability with respect to divergence and travelling wave flutter modes at the outermost radius of the compliant annulus. The critical wavenumber for the compliant surfaces is $\bar{\alpha}_{d}=0.2$.

The time history plotted in figure $23(d)$ for the mid-point $r=625$ of the compliant annulus indicates that the temporal growth of the disturbance is not suppressed indefinitely. The disturbance begins to behave like it would over a rigid wall and grow again at later times. It is plausible to attribute such longer-term behaviour to the finite radial extent of the compliant annulus. Over the time interval considered, the 
disturbance exhibits continual temporal growth at locations beyond the outer radius of the compliant insert, where the disc surface remains rigid. Ultimately, of course, like the case of the purely rigid disc discussed in $\S 3$, long-term temporal amplification does not persist. However, it would appear that the growth of the disturbance over the outermost portion of the disc influences the development elsewhere. Destabilizing effects are eventually transmitted, radially inwards, back to the compliant part of the disc surface. Evidence in support of such behaviour was obtained by conducting a simulation for a compliant annulus with the same inner radius and identical material properties, but a larger outer radius. The stabilization of the disturbance due to the surface compliance was then found to be both stronger and more persistent than with the previous simulation.

\section{Conclusions}

We have a carried out a study of the linear global behaviour corresponding to the absolute instability of the rotating-disc boundary layer. This study is based on direct numerical simulations of the complete linearized Navier-Stokes equations obtained with the novel velocity-vorticity method described in Davies \& Carpenter (2001). As the equations are linear they become separable with respect to the azimuthal coordinate, $\theta$. This permits us to simulate a single azimuthal mode. Impulse-like excitation is used throughout. This creates disturbances that take the form of wavepackets, initially containing a wide range of frequencies. When the real spatially inhomogeneous flow is approximated by a spatially homogeneous flow (the so-called parallel-flow approximation) the results of our simulations are fully in accordance with the theory of Lingwood (1995). If the flow parameters are such that her theory indicates convective behaviour the simulations clearly exhibit the same behaviour. And behaviour fully consistent with absolute instability is always found when the flow parameters lie within the theoretical absolute-instability region. Our numerical simulations also reproduce the behaviour seen in her experimental study (Lingwood 1996). In particular, there is close agreement between simulation and experiment for the ray paths traced out by the leading and trailing edges of the wavepackets.

In absolutely unstable regions the short-term behaviour of the simulated disturbances exhibit strong temporal growth and upstream propagation. This is not sustained for longer times, however. Our study suggests that convective behaviour dominates at all the Reynolds numbers we have investigated, even for strongly absolutely unstable regions. The trends found in the simulation data indicate that the disturbance behaviour eventually becomes convective, not just in the immediate vicinity of the point of impulsive excitation, but also at the increasingly remote radial locations that are encountered as the disturbance wavepacket propagates radially outwards. Predominantly convective behaviour could still be detected even when the initial impulse was positioned at a radius corresponding to one and a half times the critical Reynolds number for the onset of absolute instability in the homogeneous flow. In physical experiments the rotating-disc boundary layer has always been found to be turbulent at radial positions associated with such high values of the Reynolds number. Our simulations thus provide strong evidence that the absolute instability of the rotating-disc boundary layer does not produce a linear amplified global mode in the manner described by Huerre \& Monkewitz (1990) and as observed in many other flows.

In this respect, our results are in complete accord with the very recent study by Peake \& Garrett (2003) of the global linear stability of the rotating-disc boundary layer based 
on inviscid stability theory. Instead the absolute instability seems to be associated with transient temporal growth, much like an algebraically growing disturbance. The transient growth and associated upstream propagation would probably be sufficient to provide a route to transition and to fix the transition point at the experimentally observed location. However, the coherent structures in the downstream turbulent boundary layer would be convective rather than dominated by global oscillatory modes as in the cylinder wake. The maximum growth rates found for the simulated disturbances in the real spatially inhomogeneous flow are determined by the convective components and are little different in the absolutely unstable cases from the purely convectively unstable cases. In short, the picture emerging from our study is the opposite to the one described by Huerre \& Monkewitz whereby a region of absolute instability produces a global oscillator that dominates a downstream convectively unstable region. For the rotating-disc boundary layer, in contrast, it appears that a strong convective instability dominates a downstream region of absolute instability.

In addition to the study of the global behaviour for the usual rigid-walled rotating disc, we also investigated briefly the effect of replacing an annular region of the disc surface with a compliant wall. It had been shown earlier by Carpenter \& Cooper (1997b) that wall compliance strongly suppressed the absolute instability. Thus if the inboard radius of the compliant annulus were located in the region of absolute instability, it would have the effect of shrinking the region of absolute instability. Huerre \& Monkewitz (1990) and others found that in such cases when the region of absolute instability is sufficiently small, the temporal growth tends to be suppressed. In an analogous manner we found that the compliant annulus had the effect of suppressing the transient temporal growth in the inboard (i.e. upstream) absolutely unstable region. In fact, as time progressed the upstream influence of the compliant region became more extensive.

We are well aware of the various problems that can beset numerical simulations of absolutely unstable flows. Accordingly we took great care to ensure that our results were not produced by spurious numerical effects. Even so, it is almost impossible for a numerical simulation or experiment to establish the nature of the linear global behaviour beyond doubt. Our results, although reasonably convincing, need to be confirmed theoretically. This has been done very recently for inviscid instabilities, at least, by Peake \& Garrett (2003). Finally, it should be emphasized that our results do not invalidate in any way the theory or experiments of Lingwood $(1995,1996)$. Nor do they imply that absolute instability plays no role in the laminar-turbulent transition process.

The research described in this paper was carried out in part with the support of the Engineering and Physical Sciences Research Council.

\section{Appendix. Checks on the influence of the outflow boundary condition}

The simplest way of dealing with the outer radial boundary of the computational domain is to keep it well removed from all locations where the disturbances have evolved to an appreciable amplitude. This makes it unnecessary to model the behaviour of the disturbance at the outflow. Whenever possible we adopted such an approach. However, for simulations that involved extremely long time integrations, it was not feasible to ensure that the disturbance amplitude remained negligible at the outflow. Excessive computational expense would have been incurred in ensuring that the domain always extended beyond the leading radial edge of the disturbance. Thus 

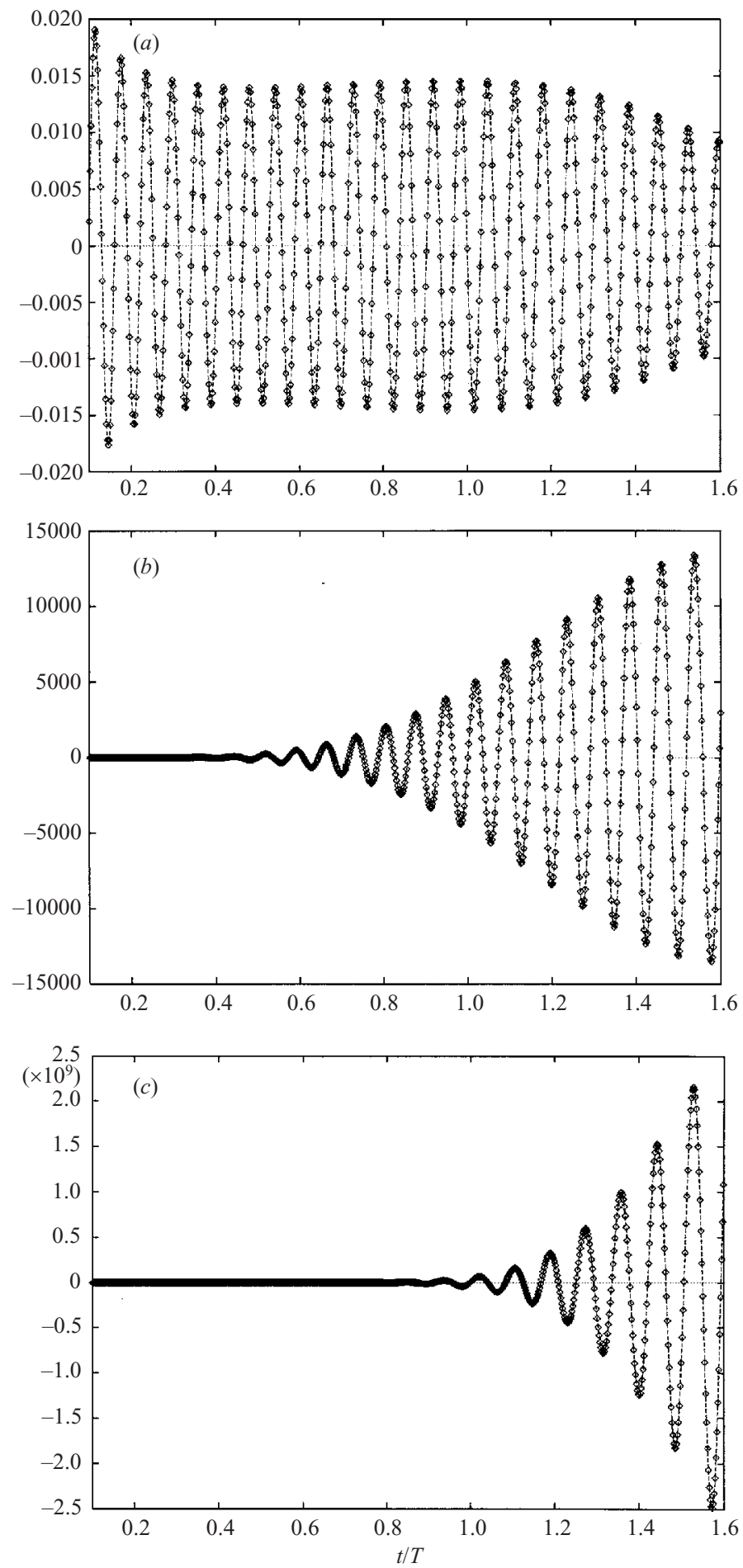

FIGURE 24. Time histories for $\omega_{\theta w}$ for a disturbance with $n=67$ that is impulsively excited at $r_{e}=530$. (a) $r=r_{e}=530 ;(b) r=630 ;$ (c) $r=730$. The data are from two numerical simulations conducted with different computational outflows located at $r_{o}=1450$ (diamonds); $r_{o}=1700$ (dashed line). 

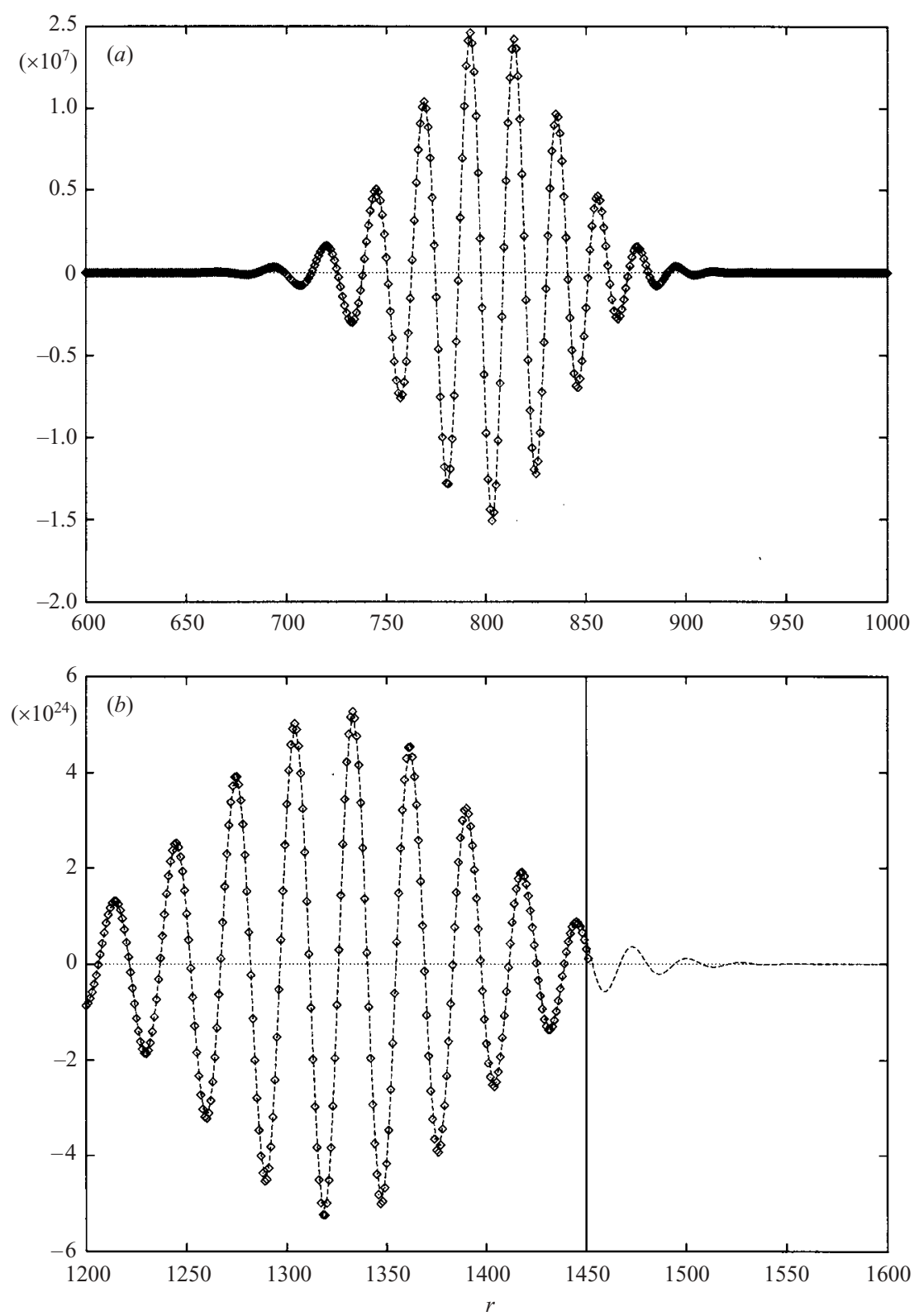

FIGURE 25. Instantaneous radial variation of $\omega_{\theta w}$ for an impulsively excited disturbance with $n=67$ and $r_{e}=530$. (a) $t / T=0.796 ;(b) t / T=1.592$. The computational outflows are again located at $r_{o}=1450$ (diamonds) (indicated in $(b)$ by a vertical line): $r_{o}=1700$ (dashed lines).

it became expedient to employ wave-like outflow boundary conditions as described in I.

As mentioned in the main part of the paper, extensive checking was undertaken to confirm that there was no contaminaton of the disturbance evolution, due to spurious transmission effects from the radial outflow boundary, in the relatively small region of interest where the behaviour of the disturbance was being subjected to detailed examination. Such checking involved the repetition of simulations using wave-like 
outflow boundary conditions that were applied at increasing radii. We illustrate this for a particular case of a disturbance with azimuthal mode number $n=67$ generated by an impulsive disk surface motion centred at $r_{e}=530$. The boundary-layer flow is taken to be spatially inhomogeneous.

Figure 24 shows the time evolution of the disturbance at successive radial locations, beginning at the radius where the disturbance was triggered. Data are presented from two different numerical simulations. For the first simulation wave-like outflow conditions are applied at $r_{o}=1450$, whereas for the second one this is increased to $r_{o}=1700$. It can be seen that there is no discernible difference in the behaviour of the disturbances over the range of radii and times considered, even though the disturbance amplitude varies by a factor of $O\left(10^{11}\right)$. In particular, it can be seen that the behaviour displayed at the centre of the impulsive excitation $r_{e}=530$ is robust. There is a period of relatively weak growth between $t / T \simeq 0.5$ and $t / T \simeq 0.9$, but otherwise the disturbance decays. Such behaviour accords with that described in the main part of the paper, where growth rates were plotted in figure 16 for the same form of disturbance.

Figure 25 displays the radial variation of the disturbance wavepacket for two selected times that are close to the middle and end of the time period considered in figure 24. Data are plotted from the two simulations conducted with different outflow boundary locations, just as before. For the first instant of time, the maximum wavepacket amplitude is located well away from the outflow boundary used in either of the simulations, so the disturbance amplitude at both outflows remains insignificant. Not surprisingly, there is no difference to be discovered in the data obtained from the two simulations. For the second selected time, it can be seen that the disturbance amplitude at the outflow boundary for the simulation conducted with the shorter computational domain has reached a significant fraction of the maximum wavepacket amplitude. This is not the case for the simulation carried out for the longer computational domain, where the amplitude of the disturbance at the outflow remains very small compared with the maximum. Nevertheless, there is still very good agreement between the results of the two simulations. There is no obvious evidence, from the radial distribution of the disturbance wavepacket, that the use of the shorter domain has led to any spurious upstream transmission effects. Returning to figure 24, it may also be noted that agreement is found in the temporal evolution of the disturbance at the point of excitation $r_{e}=530$ over the whole of the time interval considered, despite the fact that, in the simulation conducted with the shorter domain, the disturbance amplitude at the outflow $r_{o}=1450$ grows to a magnitude that is $O\left(10^{26}\right)$ times larger than the amplitude characterizing the intital impulse.

\section{REFERENCES}

BriggS, R. J. 1964 Electron-Stream Interaction with Plasmas. MIT Press.

Buell, J. C. \& Huerre, P. 1988 Inflow/outflow boundary conditions and global dynamics of spatial mixing layers. Rep. CTR-S88. Center for Turbulence Research, Stanford, USA.

CAIRns, R. A. 1979 The role of negative energy waves in some instabilities of parallel flows. J. Fluid Mech. 92, 436-450.

Carpenter, P. W., Davies, C. \& Lucey, A. D. 2000 The hydrodynamics of compliant walls: Does the dolphin have a secret? Current Sci. 79, 758-765.

Carpenter, P. W., Lucey, A. D. \& Davies, C. 2001 Status of the use of wall compliance for laminar-flow control. AIAA J. Aircraft 28, 504-512. 
CArpenter, P. W. \& Morris, P. J. 1990 The effect of anisotropic wall compliance on boundary-layer stability and transition. J. Fluid Mech. 218, 171-223.

Chomaz, J. M., Huerre, P. \& Redekopp, L. G. 1988 Bifurcations to local and global modes in spatially-developing flows. Phys. Rev. Lett. 60, 25-28.

Chomaz, J. M., Huerre, P. \& Redekopp, L. G. 1991 A frequency selection criterion in spatially developing flows. Stud. Appl. Maths 84, 119-144.

Colley, A. J., Thomas, P. J., Carpenter, P. W. \& Cooper, A. J. 1999 An experimental study of boundary-layer transition over a rotating, compliant disk. Phys. Fluids 11, 3340-3352.

COOPER, A. J. \& CARPENTER, P. W. 1997a The stability of rotating-disc boundary-layer flow over a compliant wall. Part 1. Type I and II instabilities. J. Fluid Mech. 350, 231-259.

CoOper, A. J. \& CARPenter, P. W. $1997 b$ The stability of rotating-disc boundary-layer flow over a compliant wall. Part 2. Absolute instability. J. Fluid Mech. 350, 261-270.

Cossu, C. \& Loiseleux, T. 1998 On the convective and absolute nature of instabilities in finite difference numerical simulations of open flows. J. Comput. Phys. 144, 95-108.

Davies, C. \& CARPenter, P. W. 2001 A novel velocity-vorticity formulation of the Navier-Stokes equations with applications to boundary-layer disturbance evolution. J. Comput. Phys. 172, $119-165$ (referred to herein as I).

FALLER, A. J. 1991 Instability and transition of the disturbed flow over a rotating disk. J. Fluid Mech. 230, 245-269.

Faller, A. J. \& KaYloR, R. E. 1966 A numerical study of the instability of the laminar Ekman boundary layer. J. Atmos. Sci. 23, 466-480.

GASTER, M. 1962 A note on the relation between temporally-increasing and spatially-increasing disturbances in hydrodynamic stability. J. Fluid Mech. 14, 222-224.

GASTER, M. 1965 On the generation of spatially growing waves in both space and time. J. Fluid Mech. 11, 723-727.

Gaster, M. 1968 Growth of disturbances in both space and time. Phys. Fluids 11, 723-727.

Gregory, N., Stuart, J. T. \& WALKER, W. S. 1955 On the stability of three-dimensional boundary layers with application to the flow due to a rotating disk. Phil. Trans. R. Soc. Lond. A $\mathbf{2 4 8 ,}$ $155-199$.

Hannemann, K. \& Oertel, H. 1989 Numerical simulation of the absolutely and convectively unstable wake. J. Fluid Mech. 199, 55-88.

Huerre, P. \& Monkewitz, P. A. 1990 Local and global instabilities in spatially developing flows. Annu. Rev. Fluid Mech. 22, 473-537.

Hunt, R. E. \& Crighton, D. G. 1991 Instability of flows in spatially developing media. Proc. $R$. Soc. Lond. A 435, 109-128.

Kaiktsis, L., Karniadakis, G. E. \& OrszaG, S. A. 1996 Unsteadiness and convective instabilities in two-dimensional flow over a backward-facing step. J. Fluid Mech. 321, 157-187.

KÁRmÁn, Th. von 1921 Über laminare und turbulente Reibung. Z. Angew. Math. Mech. 1, 233-252.

Kobayashi, R., Kohama, Y. \& TaKamadate, СH. 1980 Spiral vortices in boundary layer transition regime on a rotating disk. Acta Mechanica 35, 71-82.

KосH, W. 1985 Local instability characteristics and frequency determination of self-excited wake flows. J. Sound Vib. 99, 53-83.

LE Dizès, S. 1997 Global modes in falling capillary jets. Eur. J. Mech. B: Fluids 16, 761-778.

Le Dizès, S., Huerre, P., Chomaz, J.-M. \& Monkewitz, P. A. 1996 Linear global modes in spatially developing media. Phil. Trans. R. Soc. Lond. A 354, 169-212.

Leu, T.-Z. \& Ho, C.-M. 2000 Control of global instability in a non-parallel near wake. J. Fluid Mech. 404, 345-378.

LingWOOD, R. J. 1995 Absolute instability of the boundary layer on a rotating disc. J. Fluid Mech. 299, 17-33.

LINGWOOD, R. J. 1996 An experimental study of absolute instability of the rotating-disk boundarylayer flow. J. Fluid Mech. 314, 373-405.

LINGWOOD, R. J. $1997 a$ On the application of the Briggs' and steepest-descent methods to a boundary-layer flow. Stud. Appl. Maths 98, 213-254.

LINGWOOD, R. J. $1997 b$ Absolute instability of the Ekman layer and related rotating flows. J. Fluid Mech. 331, 405-428.

MACK, L. M. 1985 The wave pattern produced by a point source on a rotating disk. AIAA Paper 85-0490. 
Malik, M. R. \& Balakumar, P. 1992 Nonparallel stability of rotating disk flow using PSE. In Instability, Transition \& Turbulence (ed. M. Y. Hussaini, A. Kumar \& C. L. Streett), pp. 168-180. Springer.

Mathis, C., Provansal, M. \& Boyer, L. 1984 The Bénard-von Kármán instability: an experimental study near the threshold. J. Phys. (Paris) Lett. 45, 483-491.

Maxworthy, T. 1999 The flickering candle: transition to a global oscillation in a thermal plume. J. Fluid Mech. 390, 297-323 (and Corrigendum 399, 377).

Monkewitz, P. A. 1988 The absolute and convective nature of instability in two-dimensional wakes at low Reynolds numbers. Phys. Fluids 31, 999-1006.

Monkewitz, P. A., Huerre, P. \& Chomaz, J.-M. 1993 Global linear stability analysis of weakly non-parallel shear flows. J. Fluid Mech. 251, 1-20.

Monkewitz, P. A. \& NGuYen, L. N. 1987 Absolute instability in the near-wake of two-dimensional bluff bodies. J. Fluids Struct. 1, 165-184.

Oertel, H. 1990 Wakes behind blunt bodies. Annu. Rev. Fluid Mech. 22, 539-564.

Peake, N \& Garrett, S. J. 2003 On the global stability of the boundary layer on rotating bodies. Submitted to J. Fluid Mech.

Pier, B. \& Huerre, P. 1996 Fully nonlinear global modes in spatially developing media. Physica D 97, 206-222.

Pier, B., Huerre, P., Chomaz, J.-M. \& Couairon, A. 1998 Steep nonlinear global modes in spatially developing media. Phys. Fluids 10, 2433-2435.

Pierrehumbert, R. T. 1984 Local and global baroclinic instability of zonally varying flow. J. Atmos. Sci. 41, 2141-2162.

Reed, H. L. \& Saric, W. S. 1989 Stability of three-dimensional boundary layers. Annu. Rev. Fluid Mech. 21, 235-284.

Saric, W. S., Reed, H. L. \& White, E. B. 2003 Stability and transition of three-dimensional boundary layers. Annu. Rev. Fluid Mech. 35, 413-440.

Schër, C. \& Smith, R. B. 1993 Shallow-water wave flow past isolated topography. Pt. II: Transition to vortex shedding. J. Atmos. Sci. 50, 1401-1412.

Schlichting, H. 1979 Boundary-Layer Theory, 7th Edn, pp. 102-104. McGraw-Hill.

Spalart, P. R. 1991 On the cross-flow instability near a rotating disk. Proc. Boundary Layer Transition \& Control, pp. 22.1-13. Royal Aero. Soc.

Strykowski, P. J. \& Niccum, D. L. 1991 The stability of countercurrent mixing layers in circular jets. J. Fluid Mech. 227, 309-343.

Strykowski, P. J. \& SReenivasan, K. R. 1990 On the formation and suppression of vortex shedding at low Reynolds number. J. Fluid Mech. 218, 71-107.

Triantafyllou, G. S. \& Dimas, A. 1989 The interaction of two-dimensional flows with a free surface at low Froude Numbers. Phys. Fluids A 1, 1813-1821.

Triantafyllou, G. S., Triantafyllou, M. S. \& Chryssostomidis, C. 1986 On the formation of vortex streets behind stationary cylinders. J. Fluid Mech. 170, 461-477.

Turkyilmazoglu, M. \& GajJar, J. S. B. 2000 Direct spatial resonance in the laminar boundary layer due to a rotating-disk. Sādhanā - Acad. Proc. Engng Sci. 25, 601-617.

Zielinska, J. A. \& WestFried, J. E. 1995 On the spatial structure of global modes in wake flow. Phys. Fluids 7, 1418-1424. 